\title{
Level statistics across the many-body localization transition
}

\author{
Piotr Sierant \\ Instytut Fizyki imienia Mariana Smoluchowskiego, Uniwersytet Jagielloński, \\ ulica Profesora Stanisława Lojasiewicza 11, PL-30-348 Kraków, Poland \\ Jakub Zakrzewski \\ Instytut Fizyki imienia Mariana Smoluchowskiego, Uniwersytet Jagielloński, \\ ulica Profesora Stanistawa Eojasiewicza 11, PL-30-348 Kraków, Poland and \\ Mark Kac Complex Systems Research Center, Uniwersytet Jagielloński, \\ ulica Profesora Stanistawa Eojasiewicza 11, PL-30-348 Kraków, Poland
}

(Dated: March 6, 2019)

\begin{abstract}
Level statistics of systems that undergo many-body localization transition are studied. An analysis of the gap ratio statistics from the perspective of inter- and intra-sample randomness allows us to pin point differences between transitions in random and quasi-random disorder, showing the effects due to Griffiths rare events for the former case. It is argued that the transition in the case of random disorder exhibits universal features that are identified by constructing an appropriate model of intermediate spectral statistics which is a generalization of the family of short-range plasma models. The considered weighted short-range plasma model yields a very good agreement both for level spacing distribution including its exponential tail and the number variance up to tens of level spacings outperforming previously proposed models. In particular, our model grasps the critical level statistics which arise at disorder strength for which the inter-sample fluctuations are the strongest. Going beyond the paradigmatic examples of many-body localization in spin systems, we show that the considered model also grasps the level statistics of disordered Bose- and Fermi-Hubbard models. The remaining deviations for long-range spectral correlations are discussed and attributed mainly to the intricacies of level unfolding.
\end{abstract}

\section{INTRODUCTION}

It is 90 years already since Wishart in a seminal paper [1] introduced the concept of random matrices into science. His original aim was to generalize the chi-squared distribution to multiple dimensions, random symmetric non negative matrices played then the role of random variables. The corresponding Wishart distribution found many applications from modern random matrix theory [2] to various applications in physics [3-6], wireless communications [7] financial data for large portfolios [8] etc.

The next big step came with the introduction of Gaussian ensembles and the realization of Wigner and others [9] that spectra for usually unknown complex nuclear Hamiltonians may be understood statistically using properties of these ensembles obeying appropriate symmetries. It became a textbook knowledge that there exist exactly three universality classes $[10,11]$ the Gaussian Orthogonal Ensemble (GOE) corresponds to systems invariant with respect to (generalized) time-reversal, the Gaussian Unitary Ensemble corresponds to systems with broken time-reversal invariance and the symplectic ensemble corresponds to half-integer spin systems with preserved time-reversal invariance and no other symmetries present. Thus, since the sixties it was the common knowledge that spectra of many-body interacting systems are statistically well described by random matrix theory (RMT). Further justifications of successes of RMT

\footnotetext{
* jakub.zakrzewski@uj.edu.pl
}

come from the theory of Dyson yielding the gaussian ensembles from an appropriate statistical mechanics description [12-15].

An interesting development appeared in the eighties - the conjecture that statistical properties of spectra of systems chaotic in the classical limit are faithful to random matrix predictions [16]. This came as a surprise even simple single particle Hamiltonians containing no randomness and represented by large, very sparse (due to strong selection rules in appropriately chosen basis) matrices were statistically faithful to RMT predictions as revealed e.g. in the study of hydrogen atom spectrum in the presence of strong magnetic field inducing the so called quadratic Zeeman coupling [17]. More precisely, after unfolding the levels (obtaining the mean density of states equal to unity) the remaining fluctuations were faithfully represented by predictions of RMT [18] as shown by nearest neighbor spacing distribution, $P(s)$, the so called number variance (i.e. the variance of the number of levels in an interval of length $L$ ), correlation functions etc. The same measures indicated, however, that the transition from the chaotic to integrable situation (described by Poisson ensemble of uncorrelated levels for systems of large dimensions [18]) seems system specific and determined by the structure of the underlying classical mechanics in the mixed phase space [19].

Similar transition from extended to localized states as revealed e.g. by a change of level statistics from GOElike to Poisson-like appears in the Anderson localization transition. The corresponding level statistics has been addressed in the seminal paper [20] followed by other important developments [21-23] to mention early contri- 
butions - for a review see [24]. In those cases a single particle problem in disordered medium was addressed.

Recent years provided another important example of such a transition between ergodic (describable by standard gaussian RMT) and integrable limits - the manybody localization (MBL). While for weak disorder manybody interacting systems behave as expected for a long time being ergodic and following gaussian RMT predictions, for a sufficiently strong disorder a gradual (for finite systems sizes) crossover to localized situation occurs [25]. This phenomenon attracted an enormous interest in the last 10 years as it provides a robust example of non ergodic behavior in a complex many-body system. Instead of effectively thermalizing (as suggested by the eigenvector thermalization hypothesis (ETH) [26]) such a strongly disordered systems often remember their initial state as manifested in a series of spectacular experiments [27-29]. Already early theoretical studies [30] showed that a transition to MBL situation is accompanied by a change of level statistics from that corresponding to GOE to Poisson-like for MBL.

Importantly, it has been suggested that MBL phase is indeed integrable $[31,32]$, namely in MBL phase a complete set of local integrals of motions (LIOMS) may be defined. On one side finding LIOMs provides information about the system for a given disorder realization (LIOMs are disorder realization dependent) - on the other side the very existence of LIOMs explains the Poissonian statistics observed deep in the localized phase. While the two extremal situations - the metallic, GOE-like ergodic behavior for a weak disorder and the full MBL phase seem to be presently quite well understood it is desirable to understand and describe the nature of the ergodic-MBL transition.

The problem is not simple - it has been found, in particular, that the nature of the disorder plays a decisive role in the character of the transition [33, 34]. Intrasample randomness was indentified as the dominant feature for quasiperiodic disorder (QPD) while the intersample randomness is an essential property of transition for purely random disorder (RD). Those important observations were made studying the entanglement entropy behavior.

In this work, we show that a proper analysis of gap ratio statistics allows us to get similar insight on the randomness of system in MBL transitions as the entanglement entropy [33]. Our method is conceptually simpler as it relies only on the spectrum of the system and as such can be straightforwardly used in studies of various complex systems. Secondly, this analysis, as a by-product, gives hints on the construction of universal model of level statistics for MBL transition which we provide generalizing earlier attempts $[20,35,36]$. We introduce a weighted short-range plasma model (wSRPM) and argue that it describes faithfully the level statistics during the whole crossover between ergodic and MBL phases at system sizes accessible in exact diagonalization studies. Taking into account the inter-sample randomness - an inherent feature of MBL transition in systems with random disorder, the proposed model grasps correctly not only the bulk properties of the level spacing distribution $P(s)$ but also its exponential tails and correctly reproduces the number variance, $\Sigma^{2}(L)$, at $L$ of the order of tens level spacings. This implies that wSRPM reflects faithfully both short-range and long-range spectral correlations in systems across the ergodic-MBL crossover. Remaining small discrepancies are discussed in details providing a further insight into the long range spectral correlations of the system. Furthermore, we show that the wSRPM is universal as it works across the whole ergodic to MBL crossover not only in spin models but also in disordered bosonic and fermionic systems. We compare our results with earlier propositions [35-39] showing that the model proposed by us represents the data much more faithfully. We also discuss an alternative model of level statistics - weighted power-law random banded matrix model, which also accurately grasps spectral correlations across the ergodic-MBL crossover.

\section{GAP RATIO ANALYSIS}

A dimensionless ratio of consecutive energy levels gaps (referred as the gap ratio) was introduced in [30]. It is defined as $r_{n}=\min \left\{\delta_{n}, \delta_{n-1}\right\} / \max \left\{\delta_{n}, \delta_{n-1}\right\}$ where $\delta_{n}=E_{n+1}-E_{n}$ is an energy difference between two consequtive levels. The average gap ratio, $\bar{r}$, is different for systems with extended eigenstates (in the following we shall concentrate on the gaussian orthogonal ensamble (GOE) for time-reversal invariant systems) $\bar{r}_{G O E} \approx 0.53$ and for localized systems $\bar{r}_{P o i} \approx 0.39$ as was analyticaly demonstrated in [40]. That property was used by many authors in attempts to localize the MBL transition [30, 41-49].

The usual way of calculating the mean gap ratio $\bar{r}$ is to average the $r_{n}$ variable over a certain number of energy levels getting a mean gap ratio for one sample $r_{S}=\left\langle r_{n}\right\rangle_{S}$. Then, the mean gap ratio is obtained by averaging of $r_{S}$ over disorder realizations $\bar{r}=\left\langle r_{S}\right\rangle_{d i s}$. While, as mentioned above $\bar{r}$ obtained in this way reflects the character of eigenstates of the system [30, 41-45, 47] a part of information encoded in the $r_{n}$ variables is necessarily lost. Let us examine $P\left(r_{S}\right)$ - the distribution of the sample averaged gap ratio $r_{S}$ - it provides a direct information about variations of the $r_{S}$ for different disorder realizations. As an example we consider the XXZ spin- $1 / 2$ chain with additional next-nearest-neighbors coupling (similar to that of [33])

$H=J \sum_{i=1}^{K} \vec{S}_{i} \cdot \vec{S}_{i+1}+W \sum_{i=1}^{K} \cos (2 \pi \zeta i+\phi) S_{i}^{z}+J_{1} \sum_{i=1}^{K} S_{i}^{z} S_{i+2}^{z}$,

where $\vec{S}_{i}$ are spin- $1 / 2$ matrices, $\zeta=(\sqrt{5}-1) / 2$ (the golden ratio) and $\phi$ is a fixed phase for a given disorder realization (leading to QPD) or is random on each lattice site (leading to RD with the same on-site distribution, 

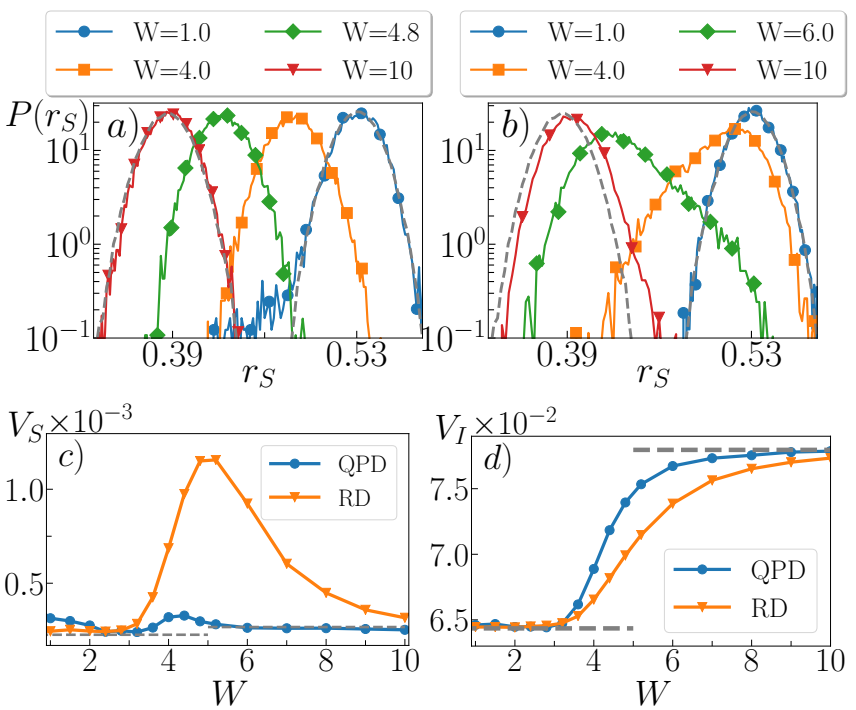

Figure 1. Top: (a) the distributions $P\left(r_{s}\right)$ for quasi-periodic disorder (QPD) of strength $W$. Dashed lines give limiting GOE and Poisson behaviors. The tail of the $W=1$ distribution indicates that QPD reproduces GOE statistics only approximately; (b) the distributions $P\left(r_{s}\right)$ for random disorder (RD). Bottom: (c) the inter-sample variance $V_{S}$ for $\mathrm{RD}$ and QPD; (d) the intra-sample variance $V_{I}$ for QPD and RD during the transition.

as in the QPD case) [33]. We fix $J=1$ as the energy unit and we study the case of $J_{1}=J$ first. Periodic boundary conditions are assumed so that $\vec{S}_{K+1}=\vec{S}_{1}$. For the system size $K=16$ we consider sequences of $N=400$ consecutive eigenvalues from the middle of the spectrum yielding a collection of $r_{S}$ values for $n_{\text {dis }}=2000$ disorder realizations. The resulting distributions, $P\left(r_{S}\right)$, for different disorder strengths $W$ are shown in Fig. 1.

Had all $r_{n}$ been independent of each other the distribution of $r_{S}=\sum_{n=1}^{N} r_{n} / N$ should be Gaussian with width determined by the variance of the $r_{n}$ distribution and proportional to $1 / \sqrt{N}$. Despite the correlations - particularly strong for GOE - the $P\left(r_{S}\right)$ are Gaussian in the limiting cases of GOE and Poisson statistics. Surprisingly, the $P\left(r_{S}\right)$ distributions remain Gaussian for QPD across the transition.

In a striking contrast, the distributions in the RD case become strongly asymmetric with enlarged variance in the transition region. This reflects the inter-sample randomness importance for the RD and is a clear, nice manifestation of the existence of rare Griffiths regions [50-53]: for samples with $\bar{r}$ close to GOE there exist realizations of disorder leading to $r_{S}$ close to Poisson limit. Similarly, on a localized side for $\bar{r}$ close to integrable limit there are rare events with $r_{S}$ values close to GOE value. The stark difference in the $P\left(r_{S}\right)$ distributions between the RD and QPD cases can be quantified by calculating a variance: $V_{S}=\left\langle r_{S}^{2}-\bar{r}^{2}\right\rangle_{d i s}$. As Fig. 1c) shows, the inter-sample variance $V_{S}$ has a clear peak in the MBL transition for the $\mathrm{RD}$ whereas it varies only slightly for the QPD.
Consider now the variance $v_{I}$ of the $r_{S}$ variable, $v_{I}=\left\langle r_{n}^{2}-r_{S}^{2}\right\rangle_{S}$. Averaged over disorder realizations $V_{I}=\left\langle v_{I}\right\rangle_{d i s}$, it provides information about fluctuations of $r_{n}$ within a single spectrum of the system at a certain disorder strength - characterizing intra-sample randomness. As could be expected from the long range correlations of GOE, it is small for GOE and conversely, it is maximal for Poissonian spectrum. Fig. 1d) shows that it behaves similarly for QPD and RD interpolating between the values for GOE and Poisson statistics. The transition is sharper for the system with QPD, implying that it is less affected by finite size effects [33].

Seeing that the distribution $P\left(r_{S}\right)$ and the variances $V_{S}$ and $V_{I}$ provide a valuable information about the randomness at the MBL transition, let us switch our attention to the more standard Heisenberg chain case taking $J_{1}=0$ in Eq. (1) and assuming random uniform disorder so that $W \cos (2 \pi \zeta i+\phi)$ is exchanged by $h_{i} \in[-W, W]$ in Eq. (1), explicitly

$$
H=J \sum_{i=1}^{K} \vec{S}_{i} \cdot \vec{S}_{i+1}+\sum_{i=1}^{K} h_{i} S_{i}^{z} .
$$

Despite the fact that the distribution of disorder is different and the studied model contains now nearest neighbor couplings only, the $P\left(r_{S}\right)$ behaves quite similarly to the case shown in Fig. 1b) revealing strong asymmetry and broadening across the transition - as shown in Fig. 2. Particularly, the broader distributions in the transition regime suggest that one may use the maximal variance $V_{S}$ as an indicator of the transition point.

A standard finite size scaling of different quantities can be performed assuming $W \rightarrow\left(W-W_{C}\right) K^{1 / \nu}$. For $\bar{r}$ such an analysis has been performed already $[43,54]$ with the data collapsing to a single curve. Similar scaling may be used for the variance $V_{S}$. Observe that both the position of the maximum as well as its value depend on the system size - Fig. 2a). If, together with the rescaling of the disorder strength, the variance $V_{S}$ is rescaled according to $V_{S} \rightarrow \widetilde{V}_{S}=\left(V_{S}-V_{G O E}\right) / K^{\kappa}$ (where $V_{G O E}$ is the inter-sample variance for GOE) the data for various system sizes collapse onto a single curve - Fig. 2c) for the exponents $\nu=0.95(10), \kappa=1.2(1)$ and the critical disorder strength $W_{C}=3.5(1)$. The scaling of the $V_{S}$ will necessarily cease to work for larger system sizes as the support of the $P\left(r_{S}\right)$ distribution is limited by $\bar{r}_{P o i}$ and $\bar{r}_{G O E}$. On the other hand, the critical disorder strength $W_{C}=3.5(1)$ and the exponent $\nu=0.95(10)$ are in nice agreement with results of [43]. A similar finite size scaling may be performed for the intra-sample variance $V_{I}$ with the same $W_{C}$ and $\nu$ - Fig. 2d). It is notable that all three measures $\bar{r}, V_{S}$ and $V_{I}$ scale in a very similar manner. Being interconnected they still provide different insights into physics of the system during the MBL transition.

The gap ratio analysis demonstrates that more than just an overall information about the crossover between ergodic and MBL regimes can be obtained from the $r_{n}$ 

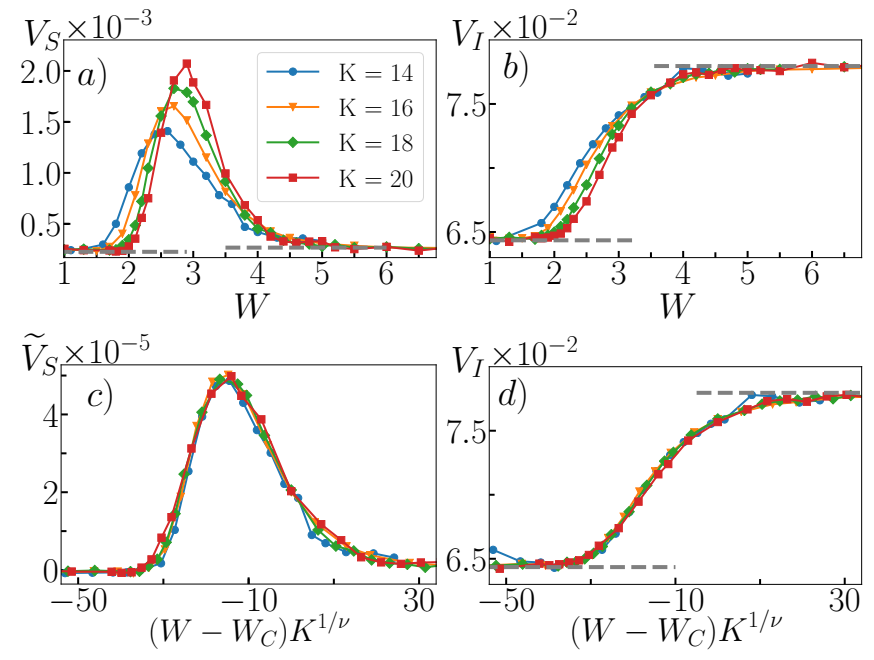

Figure 2. Top: (a) The variance $V_{S}$ of the $r_{S}$ distribution characterizing the inter-sample randomness; (b) the variance $V_{I}$ reflecting the intra-sample fluctuations in the spectrum of the system. Bottom: (c) the rescaled inter-sample variance $\widetilde{V}_{S}$ and (d) the intra-sample variance $V_{I}$ collapse after the rescaling of the disorder strength with $W_{C}=3.5$ and $\nu=$ 0.95. Data for system sizes $K \in\{14,16,18,20\}$.

variables. The considered inter- and intra-sample variances $V_{S}$ and $V_{I}$ reflect nicely the differences between RD and QPD universality classes. Furthermore, the $P\left(r_{S}\right)$ distribution quantifies the inter-sample fluctuations of a system undergoing MBL transition and gives a particularly clear demonstration of the Griffiths regime.

Moreover, the gap ratio analysis hints how to formulate the wSRPM model of spectral statistics across the MBL transition for the random disorder - namely, the ensemble we are looking for should take into account the large inter-sample randomness of the RD case. The problem of construction of such an ensemble will be considered in the remaining part of the manuscript. We start by reviewing the existing models of level statistics in the MBL transition.

\section{LEVEL STATISTICS IN MBL TRANSITION}

A number of models for intermediate statistics in the MBL transition have been proposed in [35, 36, 38, 39]. In this section we compare level spacing distributions $P(s)$ and number variances $\Sigma^{2}(L)$ predicted by those models with data for the standard model of MBL - XXZ spin- $1 / 2$ chain Eq. (2), studied already in the previous section. As we have seen this model undergoes a transition to MBL at $W_{C} \approx 3.5$ in the thermodynamic limit $K \rightarrow \infty$, in the center of the spectrum $\left(W_{C} \approx 3.7\right.$ was obtained in [43]). Fig. 3 shows level spacing distribution $P(s)$ and number variance $\Sigma^{2}(L)$ for the system (2) of size $K=16$ at disorder strength $W=1.9$ compared with predictions of different proposed models $[35,38,39]$ of the flow of level statistics between GOE and PS limits supplemented by data for the short-range plasma model (SRPM) [55]. The numerical data for spacing distribution and the number variance for XXZ spin chain are fitted with those models. Mean field plasma model. The work [35] describes the flow of level statistics across the MBL transition. Close to the ergodic regime a mean field plasma model [21] with an effective power-law interaction between energy levels is proposed. It predicts the level spacing distribution and the number variance to be

$$
P(s)=C_{1} s^{\beta} \mathrm{e}^{-C_{2} s^{2-\gamma}} \text { and } \quad \Sigma_{2}(L) \propto L^{\gamma}
$$

with $C_{1,2}$ determined by normalization conditions $\langle 1\rangle=$ $\langle s\rangle=1$. The exponents $\beta, \gamma$ reflect a local repulsion of energy levels and an effective range of interactions between energy levels. They are treated as fitting parameters which vary across the transition. Note that for $\gamma=1$ the eigenvalues are interacting only locally leading to semi-Poisson statistics

$$
P(s) \propto s^{\beta} \mathrm{e}^{-(\beta+1) s} \quad \text { and } \quad \Sigma_{2}(L) \propto \frac{1}{\beta+1} L .
$$

Between GOE and Poisson limits the exponent $\gamma$ satisfies $0<\gamma<1$. It follows from (3) that tail of the level spacing distribution decays faster than exponentially with $s$ and that the number variance $\Sigma^{2}(L)$ increases as a power law of $L$. The level spacing distribution and the number variance predicted by this model are denoted by the solid violet line in Fig. 3 - the values of $\beta$ and $\gamma$ are obtained by the least square fit to the bulk of $P(s)$ and the multiplicative factor in front of the $\Sigma^{2}(L)$ is treated as the third fitting parameter. While the bulk of the level spacing distribution is nicely recovered, the tail of the $P(s)$ distribution and the number variance are clearly not matching the data for $W=1.9$.

This two features were shown to be not obeyed by a system across the MBL crossover in [36] where it was demonstrated that the level spacing distributions decay exponentially with $s$. At the same time the number variance increases as $L^{\gamma}$ with $\gamma>1$ close to the ergodic phase and as the system becomes more localized it becomes asymptotically linear for large $L$.

Rosenzweig-Porter ensemble. Another work [38] suggest that Rosenzweig-Porter (RP) ensemble can be appropriate to describe the MBL transition. Multifractal properties of eigenvectors of this model, which is defined as an ensemble of real symmetric (for $\beta=1$ orthogonal class relevant for us) random matrices $M=\left(M_{i j}\right)$ of size $n \times n$ with matrix elements being independent Gaussian variables with zero average values $M_{i j}=0$ and

$$
\left\langle M_{i i}^{2}\right\rangle=1, \quad \text { and } \quad\left\langle M_{i j}^{2}\right\rangle=\sigma / 2
$$

were studied in [56]. The dotted line in Fig. 3 shows the obtained level spacing distribution and the number variance which fits best the data for the XXZ spin chain at $W=1.9$. The presented data are for $n=3000$ and 

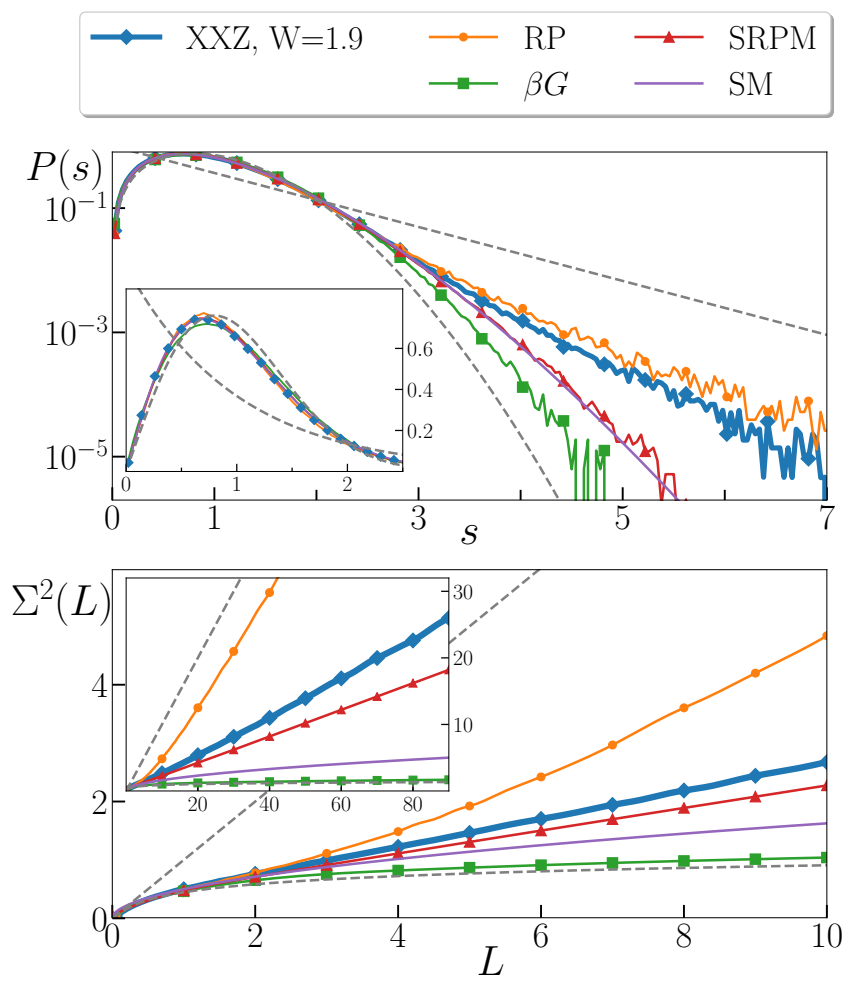

Figure 3. Top: level spacing distribution $P(s)$ for XXZ spin chain (2) of size $K=16$ at disorder strength $W=1.9$ compared with predictions of various models of the flow of level statistics between GOE and PS limits in the MBL crossover discussed in the main text. The vertical axis of the main plot is logarithmic to enable the comparison of tails of the distributions, inset shows the data in doubly linear scale; RP the Rosenzweig-Porter model at $\sigma=0.0016 ; \beta G-\beta$ Gaussian ensemble with $\beta=0.81$; SRPM - short range plasma model with the range of interactions $h=5$; SM - the mean field model (3). Bottom: the number variance $\Sigma^{2}(L)$ for the same system, inset shows the long range behavior of $\Sigma^{2}(L)$ which encodes long range spectral correlations of eigenvalues. Gray dashed lines correspond to level spacing distributions and number variances for GOE and PS limits.

$\sigma=0.0016$ and are in rather poor agreement even regarding the bulk of the $P(s)$ distributions. Moreover, at $L \gtrsim 3$ the number variance bends abruptly upwards - a feature which we do not observe for the $W=1.9$ data. Similar trend persists at larger disorder strengths $W$ indicating that one cannot reproduce both the level spacing distribution and the number variance $\Sigma^{2}(L)$ of the $X X Z$ spin chain across the MBL crossover within the RP ensemble.

$\beta$-Gaussian ensemble. The two remaining models $[39,55]$ can be specified by a joint probability distribution function (JPDF) of eigenvalues. A JPDF for a Random Matrix Ensemble can be written as the probability distribution of a one-dimensional gas of classical particles with total energy $W\left(E_{1}, \ldots, E_{n}\right)$

$$
\mathcal{P}\left(E_{1}, \ldots, E_{N}\right)=Z_{N}^{-1} \exp \left(-\beta W\left(E_{1}, \ldots, E_{n}\right)\right),
$$

where $Z_{N}$ is a normalization constant and the total energy

$$
W\left(E_{1}, \ldots, E_{n}\right)=\sum_{i} U\left(E_{i}\right)+\sum_{i<j} V\left(\left|E_{i}-E_{j}\right|\right)
$$

is determined by the trapping potential $U(E)$ and interparticle interactions $V\left(\left|E-E^{\prime}\right|\right)$. For instance, for harmonic trapping potential $U(E) \propto E^{2}$, and logarithmic interactions $V\left(\left|E-E^{\prime}\right|\right)=-\log \left(\left|E-E^{\prime}\right|\right)$ and $\beta=1$ one recovers from (6) the JPDFs for GOE, for which the interactions in (6) are between all pairs of eigenvalues which reflects the long range spectral correlations of the GOE ensemble.

One way of constructing an ensemble with statistical properties intermediate between GOE and PS is to put a rational $\beta \in[0,1]$ into JPDF (6) - in such a way a $\beta$ Gaussian ensemble ( $\beta \mathrm{GE})$ arises. A recent work [39] uses $\beta \mathrm{GE}$ to describe the level spacing distribution $P(s)$ and the gap ratio distribution in the MBL transition. Setting up appropriate tridiagonal matrices [57] of size $n=10^{5}$ and diagonalizing them, we obtain $P(s)$ and $\Sigma^{2}(L)$ for this ensemble - denoted by the green line with squares in Fig. 3. The agreement of this model with XXZ numerical data in the bulk of the $P(s)$ is not perfect. The disagreement in the tail of the $P(s)$ and the number variance is even more pronounced. Long-range correlations of eigenvalues in $\beta \mathrm{GE}$ are visible in the spectral rigidity of the spectrum - for the acquired data the number variance grows only logarithmically, just like in the GOE case, in a violent disagreement with the XXZ data. Thus, contrary to statements in [39] based on short range correlations only, the $\beta \mathrm{GE}$ is not a good candidate to describe the flow of level statistics between GOE and PS regimes across the MBL transition.

Short-range plasma models. Another way of constructing intermediate level statistics is to restrict the range of the logarithmic interactions in (6) to a finite number $h$ which leads to a family of short-range plasma models (SRPMs) [55]. Consider $N \rightarrow \infty$ particles in a ring geometry $E_{0}<E_{1}<\ldots<E_{N}<E_{N+1}, E_{N+1+k}=E_{k}$ $\bmod N$ with logarithmic interaction among $h$ neighboring eigenvalues so that the JPDF is given by

$$
\mathcal{P}_{h}^{\beta}\left(E_{1}, \ldots, E_{N}\right)=Z_{N}^{-1} \prod_{i=0}^{N}\left|E_{i}-E_{i+1}\right|^{\beta} \ldots\left|E_{i}-E_{i+h}\right|^{\beta} .
$$

For integer values of $h$ and $\beta$ this model can be analytically solved yielding the level spacing distribution

$$
P_{h}^{\beta}(s)=s^{\beta} W(s) \mathrm{e}^{-(h \beta+1) s}
$$

where $W(s)$ is a polynomial. The corresponding number variance has asymptotically linear behavior:

$$
\Sigma_{h, \beta}^{2}(L) \stackrel{L \rightarrow \infty}{\longrightarrow} \frac{L}{h \beta+1} .
$$

The SRPM can be solved analytically - see Appendix I for details. While grasping the bulk of the level spacing 
distribution $P(s)$ accurately, the SRPM model does not outperform the mean field model (3-4). One still does not obtain the correct tails of the level spacing distribution $P(s)$ or the correct slope of the number variance $\Sigma^{2}(L)$ - see the line with triangles in Fig. 3.

\section{THE WEIGHTED SRPM MODEL}

The preceding section shows that the analyzed models reproduce the bulk of the level spacing distribution and hence grasp purely local correlations of eigenvalues of a system in ergodic to MBL crossover. However, when tails of the level spacing distributions as well as the number variance are considered, the differences between data for XXZ spin chain and the predictions of the models are apparent. This shows that the models do not faithfully reproduce correlations between eigenvalues on scales larger than few level spacings. The mean field plasma model, $\beta$-Gaussian ensemble and SRPMs tend to underestimate the number variance predicting stronger long-range correlations between eigenvalues than are actually observed across the MBL crossover. The opposite is true for the RP ensemble. All in all, the SRPM with its asymptotically exponentially decaying level spacing distribution and asymptotically linear number variance gives predictions closest to the data for XXZ spin chain. For that reason we choose the SRPM as a basic building block of a more complicated, weighted ensemble which, by construction, takes into account another feature of the MBL crossover - the large inter-sample randomness.

Results of Sec. II indicate that large inter-sample randomness is an inherent feature of the MBL transition in systems with purely random disorder. It manifests itself in shape of a distribution $P\left(r_{S}\right)$ of the gap ratio for a single disorder realization $r_{S}=\left\langle r_{n}\right\rangle_{S}$ which significantly broadens in the regime of MBL transition. The broadening of $P\left(r_{S}\right)$ shows that system which has predominantly ergodic features becomes more localized for certain disorder realizations - the converse statement for mostly localized system is also true. The small fraction of events for which the system is more localized than usually reveals itself in the tail of the level spacing distribution and in the number variance. For instance consider an ensemble of matrices created in such a way that with probability $1-p$ the matrix is taken from GOE and with probability $p \ll 1$ it has the Semi-Poisson level statistics $\mathcal{P}_{h=1}^{\beta=1}$. The bulk of the level spacing distribution of such an ensemble will be very close to the Wigner distribution $P_{G O E}(s)$ of the GOE matrix ensemble (as $p \ll 1)$. However, for large level spacings the distribution will be dominated by exponentially decaying tail of the level spacing distribution $P(s)$ from the small fraction of matrices with Semi-Poisson statistics. Analogously, the number variance $\Sigma^{2}(L)$ will be a sum of logarithmically growing number variance for GOE and linearly increasing number variance for Semi-Poisson statistics. Hence, it will be dominated by the latter and increase linearly

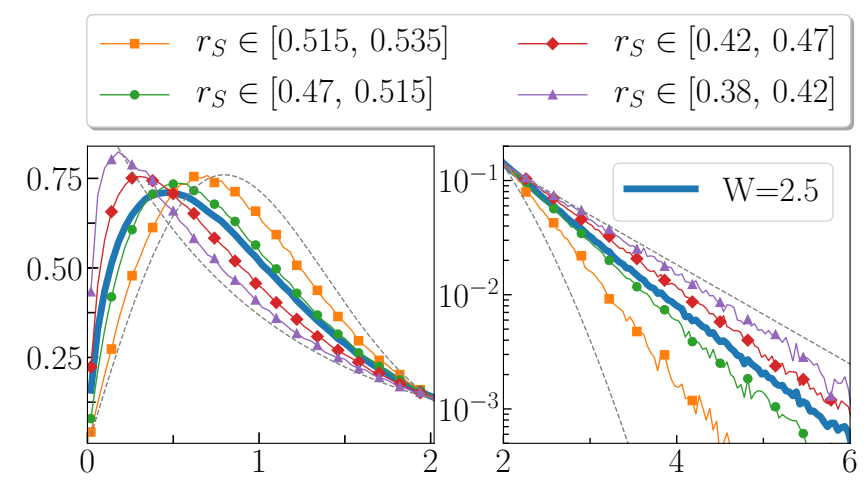

Figure 4. Level spacing distribution $P(s)$ for XXZ spin chain (2) of size $K=16$ at disorder strength $W=2.5$ (solid blue line) - left: lin-lin scale, right: lin-log scale to facilitate comparison of tails of $P(s)$. Selecting disorder realizations for which $r_{S}$ is from a given interval results in statistics with properties which vary between those of an ergodic and nearly localized system. The gray dashed lines correspond to level spacing distributions of GOE and PS.

with $L$ with a very good approximation.

This leads us to a question whether the inter-sample randomness can be responsible for the exponential tails of level spacing distribution and a linear number variance in the MBL transition via the mechanism described above. To verify this, we examine level statistics of XXZ system at certain disorder strength but accept only disorder realizations for which the $r_{S}$ belongs to a certain narrow interval - results for $W=2.5$ are presented in Fig. 4. The procedure of selecting $r_{S}$ affects significantly the resulting level statistics. As the interval of $r_{S}$ shifts towards smaller values of $r_{S}$ one obtains level spacing distributions with weaker and weaker level repulsion characterized by decreasing $\beta$ and with growing weight of the exponential tail. Both features are precisely the asymptotic characteristics of the level spacing distribution for SRPM (9) for appropriately chosen $\beta$ and $h$. However, our goal is to reproduce the full level statistics. An appropriate model should thus combine the contributions from disorder realizations with different localization properties reflected by the varying value of $r_{S}$.

This leads us to the formulation of the weighed shortrange plasma model (wSRPM) which, by definition, has JPDF given by

$$
\mathcal{P}_{g S R M P}\left(E_{1}, . ., E_{N}\right)=\sum_{i} c_{i} \mathcal{P}_{h_{i}}^{\beta_{i}}\left(E_{1}, . ., E_{N}\right)
$$

where $h_{i}$ and $\beta_{i}$ range over an appropriate set of values and $c_{i}$ are weight coefficients $\left(\sum_{i} c_{i}=1\right)$. The weight coefficients are determined by the requirement that the ${ }^{2} \mathrm{~S}-$ RPM reproduces the inter-sample randomness reflected by the $P\left(r_{S}\right)$ distribution. By integrating the JPDF for wSRPM with $\delta\left(s-\left|E_{k}-E_{k-1}\right|\right)$ one gets the level spacing distribution

$$
P_{w S R P M}(s)=\sum_{i} c_{i} P_{h_{i}}^{\beta_{i}}(s)
$$


which is a linear combination of the level spacing distributions $P_{h_{i}}^{\beta_{i}}(s)$. An analogous expression holds for the number variance

$$
\Sigma_{w S R P M}^{2}(L)=\sum_{i} c_{i} \Sigma_{\beta_{i}, h_{i}}^{2}(L),
$$

which stems from the formula $\Sigma^{2}(L)=L-\int_{0}^{L} \mathrm{~d} E(L-$ $E)\left(1-R_{2}(E)\right)$ and the fact that the two-level correlation function $R_{2}(E)$ for wSRPM is a linear combination of two-level functions of SRPMs $\mathcal{P}_{h_{i}}^{\beta_{i}}$.

\section{LEVEL SPACING DISTRIBUTION AND NUMBER VARIANCE ACROSS THE MBL CROSSOVER}

The wSRPM model, defined by (11) depends on a large number of parameters - one needs to specify JPDFs of the SRPMs $\mathcal{P}_{\beta_{i}}^{h_{i}}$ which contribute to the full JPDF of the generalized model $\mathcal{P}_{w S R P M}$ and find appropriate weight coefficients $c_{i}$. To complete this task we utilize the $P\left(r_{S}\right)$ distributions which encode the inter-sample randomness across the MBL transition. Distributions of $r_{S}$ for individual SRPMs $P_{\beta_{i}}^{h_{i}}\left(r_{S}\right)$ are Gaussian centered around $\bar{r}_{\beta_{i}}^{h_{i}}$ which depends on $h_{i}$ and $\beta_{i}$ parameters. The corresponding distribution for wSRPM reads $P_{w S R P M}\left(r_{S}\right)=\sum_{i} c_{i} P_{\beta_{i}}^{h_{i}}\left(r_{S}\right)$ and the set of parameters $\left\{h_{i}, \beta_{i}, c_{i}\right\}$ is fixed by the requirement that $P_{w S R P M}\left(r_{S}\right)$ reproduces the $P\left(r_{S}\right)$ distribution for a given physical model (in this case XXZ spin chain (2)) most faithfully. To fulfil the requirement in a robust way, we select a sequence of coefficients $\left\{\left(\beta_{i}, h_{i}\right)\right\}$ with $\bar{r}_{\beta_{i}}^{h_{i}}$ covering the interval $r_{S} \in[0.386,0.531]$ possibly uniformly, i.e. we choose

$$
\left(\beta_{i}, h_{i}\right)= \begin{cases}\left(\frac{i}{100}, 1\right), & i \in[0,10], \\ \left(\frac{i-8}{20}, 1\right), & i \in[11,30], \\ (1, i-30), & i \in\left[31,30+h_{\max }\right],\end{cases}
$$

where $h_{\text {max }}$ specifies the maximal range of interactions in the contributing SRPMs. The chosen set of coefficients (14) determines the family of wSRPM that can be obtained by various choices of the weight coefficients $\left\{c_{i}\right\}$. One way of finding the weight coefficients $\left\{c_{i}\right\}$ would be to choose a number of points $r_{j}$ and solve the linear system of equations $P\left(r_{S}=r_{j}\right)=P_{w S R P M}\left(r_{S}=r_{j}\right)$ for the $\left\{c_{i}\right\}$ coefficients given that the function $P\left(r_{S}=r_{j}\right)$ as well as $P_{\beta_{i}}^{h_{i}}\left(r_{S}\right)$ are known. If the number of the points $r_{j}$ is equal to number of coefficients $c_{i}$, the solution is unique. Unfortunately, this linear problem is badly conditioned, small changes of positions of $r_{j}$ modify drastically the solution $\left\{\tilde{c}_{i}\right\}$. In particular, it may happen that certain coefficient $\tilde{c}_{k}$ is large and positive whereas the next one $\tilde{c}_{k+1}$ is large negative which illustrates that further constraints should be imposed on $\left\{c_{i}\right\}$. Namely, the weight coefficients must be positive $c_{i}>0$ and the differences between subsequent $c_{i}$ should not be too large.

\begin{tabular}{l|ll|ll}
\hline$W$ & $\bar{r}$ & $\chi$ & $\bar{r}_{w S R P M}$ & $\chi_{w S R P M}$ \\
\hline 1.5 & 0.5306 & 0.0975 & 0.5282 & 0.078 \\
1.9 & 0.5219 & 0.259 & 0.5189 & 0.238 \\
2.1 & 0.5092 & 0.358 & 0.5074 & 0.338 \\
2.5 & 0.4720 & 0.523 & 0.4719 & 0.562 \\
\hline 2.9 & 0.4390 & 0.638 & 0.4384 & 0.711 \\
3.5 & 0.4107 & 0.774 & 0.4101 & 0.850 \\
4.5 & 0.3938 & 0.857 & 0.3941 & 0.929 \\
\hline
\end{tabular}

Table I. Values of the mean gap ratio $\bar{r}$ and spectral compressibility $\chi$ for XXZ spin chain are compared with predictions of wSRPM model: $\bar{r}_{w S R P M}$ and $\chi_{w S R P M}$.

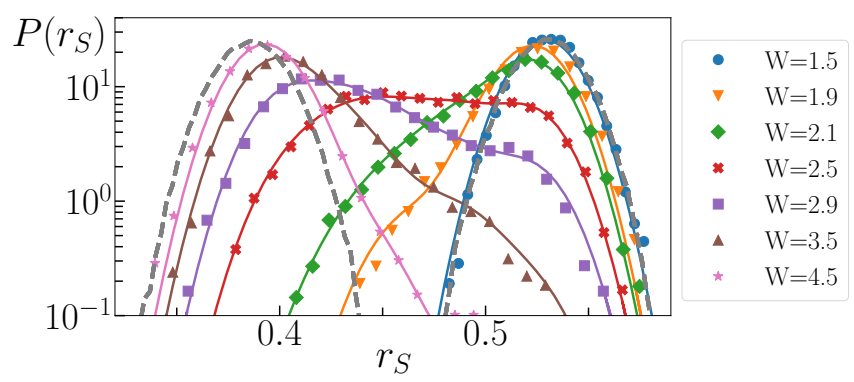

Figure 5. The fit of $P\left(r_{S}\right)$ distributions. Distributions $P\left(r_{S}\right)$ of the sample-averaged gap ratio $r_{S}$ for the XXZ spin chain Eq. (2) are denoted by markers. The corresponding wSRPM fits are denoted with solid lines. Gray dashed lines correspond to $P\left(r_{S}\right)$ for GOE and PS center respectively around $\bar{r}_{G O E}=$ 0.531 and $\bar{r}_{P S}=0.386$.

This leads us to a fitting procedure which determines the weight coefficients $\left\{c_{i}\right\}$ by minimizing

$\chi^{2}=\sum_{i=0}^{i_{\max }} \frac{1}{m}\left(c_{i+1}-c_{i}\right)^{2}+\int_{r_{S}^{m}}^{r_{S}^{M}}\left(P_{w S R P M}\left(r_{S}\right)-P\left(r_{S}\right)\right)^{2} \mathrm{~d} r_{S}$.

The term $\sum_{i=0}^{i_{\max }} \frac{1}{m}\left(c_{i+1}-c_{i}\right)^{2}$ assures the "continuity" of $c_{i}$ coefficients, the condition $c_{i}>0$ becomes a constraint of the minimization procedure. The constant $m$ is taken as $10^{-4}$ although changing it by a factor of 5 only mildly affects the results. Finally, the minimization of (15) does not resolve accurately SRPMs with large $h$ as the spacing between subsequent $\bar{r}_{\beta}^{h}$ decreases drastically, so that we take $h_{\max }=5$ in (14).

Determining the constitutive SRPMs by the choosing the set $\left\{\left(\beta_{i}, h_{i}\right)\right\}$ according to (14) and specifying the method of obtaining the weight coefficients $\left\{c_{i}\right\}$, we defined a method of finding wSRPM which reproduces inter-sample randomness and can be used across the ergodic-MBL crossover.

\section{A. Level statistics as function of disorder strength}

The distributions $P_{w S R P M}\left(r_{S}\right)$ found for the XXZ spin chain for varying disorder strength $W$ for system of size 

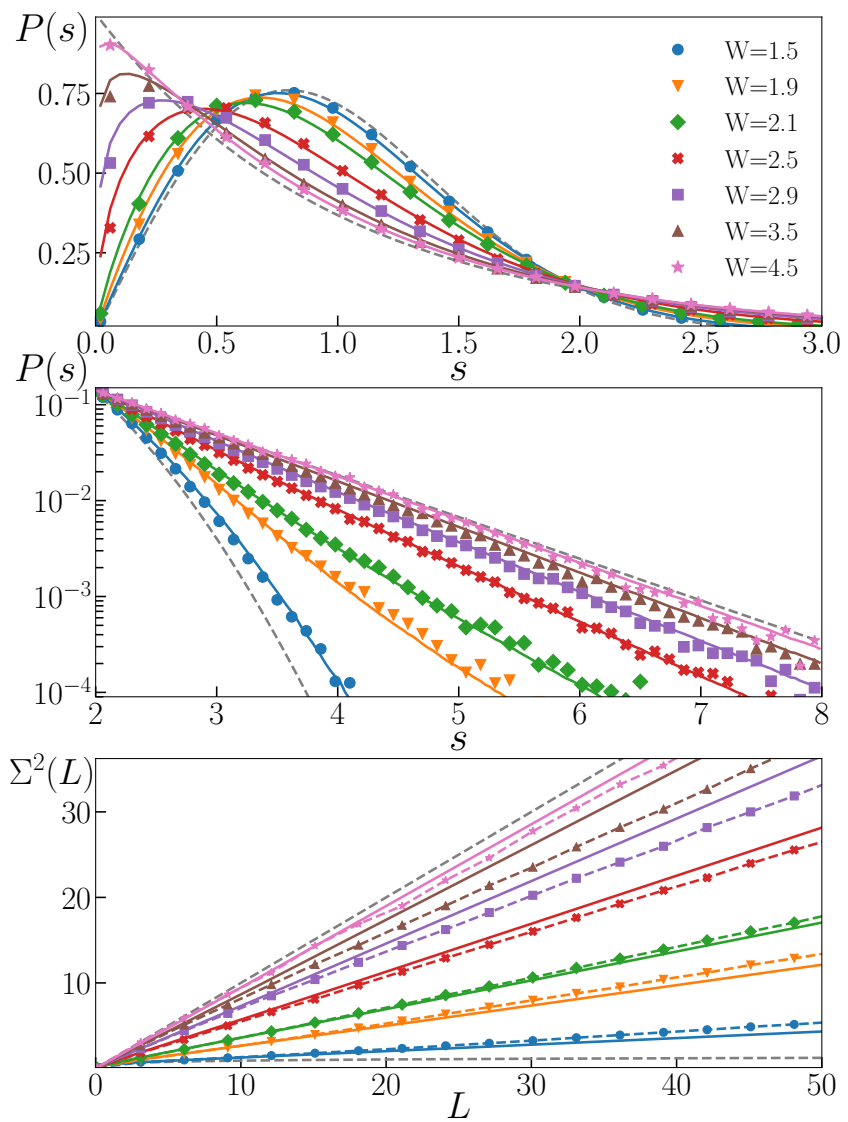

Figure 6. Top panel: level spacing distributions $P(s)$ as a function of disorder strength $W$ in XXZ spin chain (2) of size $K=16$ are denoted by markers, wSRPM results denoted by solid lines and gray dashed lines denote the level spacing distributions in the limiting GOE and PS cases; Middle panel: same as above, but vertical axis in logarithmic scale to facilitate comparison between tails of level spacing distributions; Bottom panel: dashed lines with markers - number variance $\Sigma^{2}(L)$ for XXZ spin chain, solid lines - results for wSRPM model. Gray dashed lines correspond to level spacing distribution or number variance for GOE and PS.

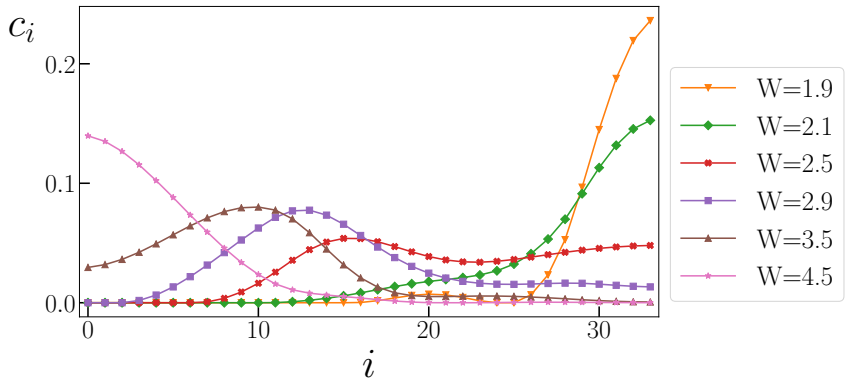

Figure 7. Coefficients $c_{i}$ for the wSRPM for XXZ spin chain across the ergodic - MBL crossover.

\begin{tabular}{l||cc|cc}
\hline & $\bar{r}$ & $\chi$ & $\bar{r}_{w S R P M}$ & $\chi_{w S R P M}$ \\
\hline$W=2.1$ & & & \\
$K=14$ & 0.4956 & 0.392 & 0.4946 & 0.432 \\
$K=16$ & 0.5092 & 0.354 & 0.5074 & 0.338 \\
$K=18$ & 0.5221 & 0.259 & 0.5193 & 0.231 \\
\hline$W=4.0$ & & & & \\
$K=14$ & 0.4021 & 0.743 & 0.4024 & 0.888 \\
$K=16$ & 0.3996 & 0.805 & 0.3992 & 0.905 \\
$K=18$ & 0.3989 & 0.849 & 0.3981 & 0.910 \\
\hline
\end{tabular}

Table II. Values of the mean gap ratio $\bar{r}$ and spectral compressibility $\chi$ for XXZ spin chain are compared with predictions of wSRPM model: $\bar{r}_{w S R P M}$ and $\chi_{w S R P M}$.

$K=16$ are presented in Fig. 5. Distributions $P\left(r_{S}\right)$ are indeed recovered in the whole crossover region. The distribution for $W=1.5$ which is already very close to the GOE regime is modeled by a single SRPM with $h=$ 12 and $\beta=1$.

Level statistics predicted by the wSRPM model together with XXZ spin chain (2) data across the MBL transition are presented in Fig. 6. We have accumulated data for $n=2000$ disorder realizations for each disorder strength $W$ and we have set the mean level spacing to unity (details described in the Sec. VII). The solid lines which denote the predictions of wSRPMs match with a very good accuracy both the bulks and the tails of level spacing distributions for disorder strengths $W$ corresponding to the whole regime intermediate between GOE and PS level statistics. In particular, the tails of $P(s)$ for $W=1.9,2.1$ are visibly bent upwards - this is a clear manifestation that SRPMs which account for more localized rare events must be included in the wSRPM. The number variances $\Sigma^{2}(L)$ predicted by the wSRPM are again reproducing the data for XXZ spin chain (2) with a very good precision. The number variance predicted by fitting of a single SRPM presented in Fig. 3 was underestimating the result for $W=1.9$ and it is the contribution from other SRPMs included in the wSRPM which ensures the agreement in the number variance.

Specific values of the weight coefficients are presented in Fig. 7. We also compare the mean gap ratio $\bar{r}$ with the prediction of wSRPM $\bar{r}_{w S R P M}=\sum_{i} c_{i} \bar{r}_{\beta_{i}}^{h_{i}}$ in Tab. I showing the agreement at the level of $0.5 \%$. In addition, we also collate spectral comprehensibilities. Predictions of wSRPM $\chi_{w S R P M}=\sum_{i} c_{i} /\left(h_{i} \beta_{i}+1\right)$ agree with spectral comprehensibilities for XXZ spin chain obtained from quadratic fit to the number variance $\Sigma^{2}(L)$ for XXZ spin chain in the interval $L \in[10,70]$ (see also Section VII) up to $10 \%$.

\section{B. Level statistics as a function of system size $K$}

We have thus demonstrated that by constructing appropriate wSRPMs one can model flow of level statistics across the ergodic-MBL crossover for XXZ spin chain at 


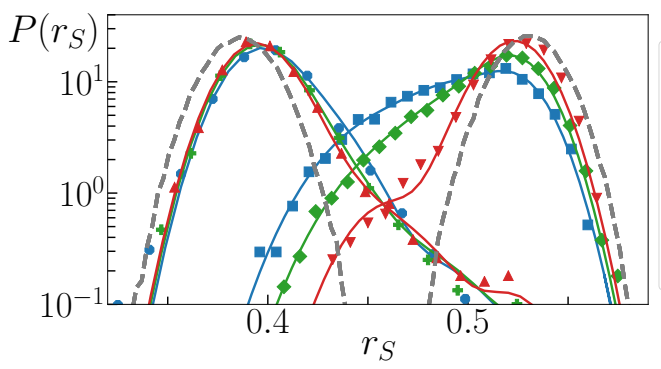

- $\mathrm{K}=14, \mathrm{~W}=2.1$

- $\mathrm{K}=14, \mathrm{~W}=4.0$

- $\mathrm{K}=16, \mathrm{~W}=2.1$

* $\mathrm{K}=16, \mathrm{~W}=4.0$

V $\mathrm{K}=18, \mathrm{~W}=2.1$

॥ $\mathrm{K}=18, \mathrm{~W}=4.0$
Figure 8. The fit of $P\left(r_{S}\right)$ distributions. Distributions $P\left(r_{S}\right)$ of the sample-averaged gap ratio $r_{S}$ for the XXZ spin chain Eq. (2) are denoted by markers. The corresponding wSRPM fits are denoted with solid lines. Gray dashed lines correspond to $P\left(r_{S}\right)$ for GOE and PS center respectively around $\bar{r}_{G O E}=$ 0.531 and $\bar{r}_{P S}=0.386$.
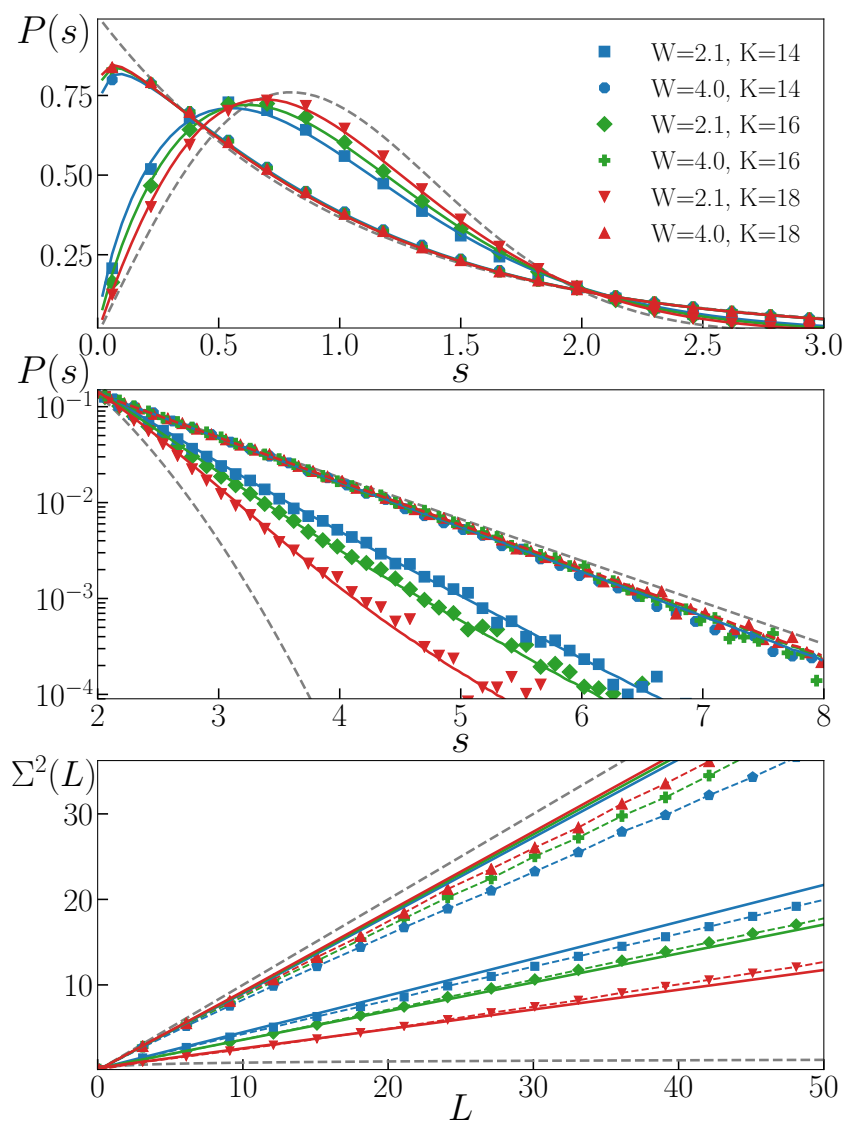

Figure 9. Top panel: level spacing distributions $P(s)$ in XXZ spin chain (2) for varying system size $K$ are denoted by markers, wSRPM results denoted by solid lines and gray dashed lines denote the level spacing distributions in the limiting GOE and PS cases; Middle panel: same as above, but vertical axis in logarithmic scale to facilitate comparison between tails of level spacing distributions; Bottom panel: dashed lines with markers - number variance $\Sigma^{2}(L)$ for XXZ spin chain, solid lines - results for wSRPM model. Gray dashed lines correspond to level spacing distribution or number variance for GOE and PS.

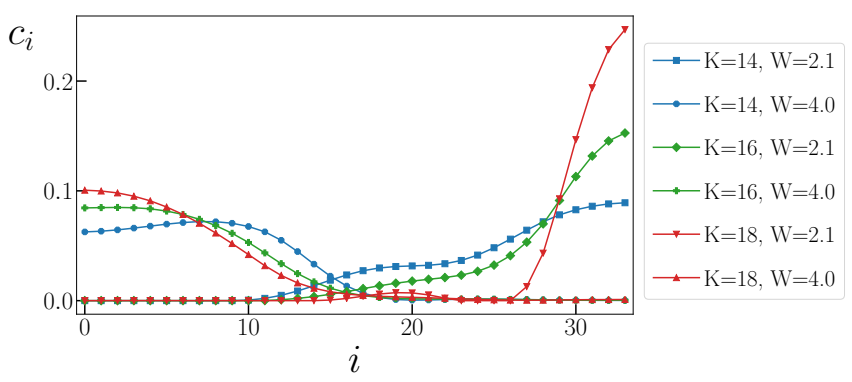

Figure 10. Coefficients $c_{i}$ for the wSRPM for XXZ spin chain for varying system size on the two sides of the ergodic - MBL crossover.

given system size. In the following subsection we demonstrate how the level statistics evolve with system size $K$ for fixed disorder strength $W$.

A quick glance at Tab. III reveals the average gap ratio $\bar{r}$ tends towards $\bar{r}_{G O E}$ and $\bar{r}_{P S}$ values with increasing system size $K$ at disorder strengths close either to the ergodic regime $(W=2.1)$ or to the MBL phase $(W=4.0)$. The system size dependence is much stronger in the former case than in the latter as it is also visible in Fig. 8. The evolution of level spacing distribution $P(s)$ and number variance $\Sigma^{2}(L)$ with increasing system size is presented in Fig. 9. The level spacing and the number variance change with increasing size as the gap ratio suggest flowing either to GOE or to PS limit. The wSRPM determined by the requirement of reproducing the the $P\left(r_{S}\right)$ distributions shown in Fig. 8 captures accurately level spacing distribution as well as the number variance for the considered system sizes. The evolution of parameters of the model with increasing system size is presented in Fig. 10 showing clearly that the weight of SRPMs closer to the GOE (those with larger $i$ ) increases with the growing system size. Analogous (albeit weaker) dependence is observed also close to the MBL regime $(W=4.0)$.

\section{Flow of level statistics in the ergodic-MBL crossover}

We have shown that wSRPM accurately describes level statistics of disordered XXZ spin chain across the whole ergodic to MBL crossover. The agreement is remarkably good even for long-range spectral correlations as shown by the number variance $\Sigma^{2}(L)$. This leads us to conclusion that the effective interactions between eigenvalues in the MBL crossover are accurately grasped by level correlations of wSRPM.

The picture of the flow of level statistics from GOE to PS in the MBL transition which emerges is the following. In the ergodic phase the range of interactions between eigenvalues tends to infinity, $h=\max \left\{h_{i}\right\} \rightarrow \infty$, and the level statistics reduces to GOE case. As the disorder strength increases, the range of interactions between eigenvalues $h$ declines to a finite value, level spac- 


\begin{tabular}{|c|c|c|c|}
\hline$K$ & $W$ & $\bar{r}$ & $\chi$ \\
\hline 14 & 2.62 & $0.4528(4)$ & $0.545(9)$ \\
\hline 16 & 2.7 & $0.4537(5)$ & $0.587(5)$ \\
\hline \multirow[t]{3}{*}{18} & 2.8 & $0.4569(7)$ & $0.605(4)$ \\
\hline & & $\bar{r}_{w S R P M}$ & $\chi_{w S R P M}$ \\
\hline & & 0.4530 & 0.639 \\
\hline
\end{tabular}

Table III. The average gap ratio $\bar{r}$ and spectral compressibility $\chi$ for the XXZ spin chain at disorder strength which corresponds to $W_{C}$ at in the thermodynamic limit $K \rightarrow \infty$. For comparison, the predictions of $\mathrm{WSRPM} r_{w S R P M}$ and $\chi_{w S R P M}$ are displayed.

ing distribution acquires an exponential tail and a finite spectral compressibility $\chi$ appears as the number variance grows linearly $\Sigma^{2}(L) \propto \chi L$. Upon further increase of the disorder strength, the range of interactions $h$ decreases further. A larger contribution of level statistics with short-range interactions appears as it is visible in tails of level spacing distributions and in the enhancement of spectral compressibility $\chi$. As the MBL phase is approached the interactions become local $h=1$ and parameter $\beta=\max \left\{\beta_{i}\right\}$ starts to flow from $\beta=1$ to $\beta=0$ in the MBL phase similarly as in the second stage of the flow described in [35]. This final stage of the flow is also accompanied by rare inclusions of systems which have nearly ergodic properties as it is visible in the $P\left(r_{S}\right)$ distribution in Fig. 5. The presence of this contribution also slightly diminishes the number variance.

Level statistics on the ergodic side of crossover flow towards GOE limit for growing system size. Similarly, for disorder strength $W \gtrsim 4.0$ system approaches the PS limit as its size increases. The transition becomes sharper and the range of disorder strengths $W$ for which a wSRPM with more than a single non-zero coefficient shrinks with increasing system size. We speculate that in the $K \rightarrow \infty$ limit only at the critical disorder strength $W_{C}$ the level statistics is neither GOE nor PS. Candidate for such critical level statistics for the MBL transition is discussed in the subsection $\mathrm{VD}$.

In conclusion, the wSRPM allows to model the level statistics in the XXZ spin chain in the whole MBL crossover. The level statistics are reproduced with a nearly perfect agreement on the level of ten level spacings. Slight discrepancies associated with long-range spectral correlations are discussed in Sec. VII. Now, we proceed to discussion of the critical level statistics in subsection VD to further demonstrate that wSRPM describes also statistics observed for other systems that reveal MBL transition in Sec. VI.

\section{Critical level statistics in MBL transition}

We assume that the critical level statistics in MBL transition can be extracted from data for a system of size
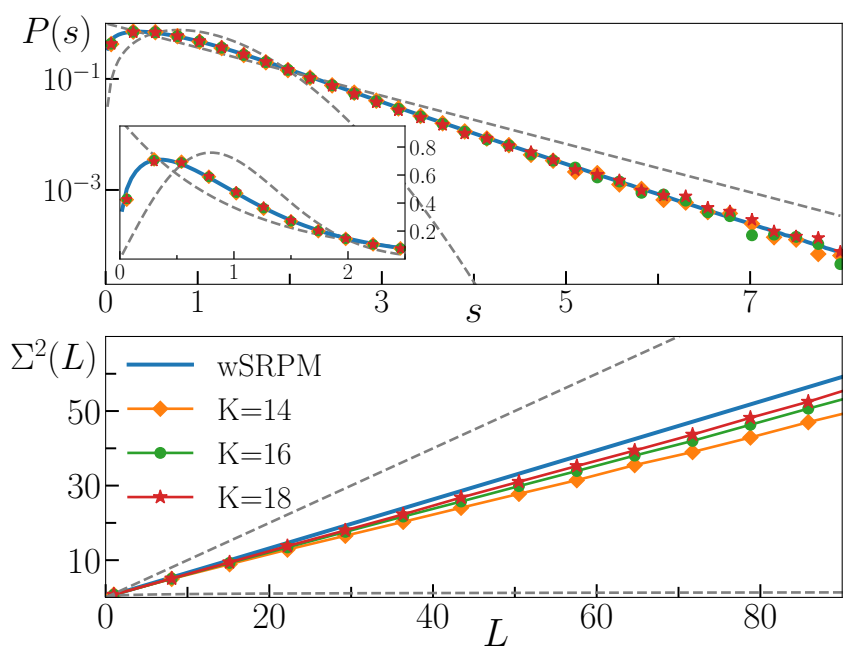

Figure 11. Critical level statistics for XXZ spin chain with random uniform disorder. Dashed lines correspond to the GOE and Poisson cases.
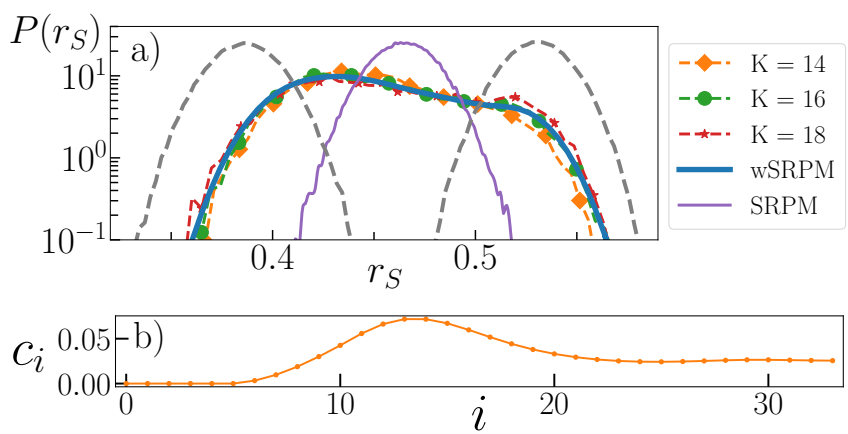

Figure 12. a) The $P\left(r_{S}\right)$ distribution for the critical statistics in XXZ spin chain along with wSRPM and $P\left(r_{S}\right)$ for SRPM. b) The $c_{i}$ coefficients of wSRPM shown in Fig 11 .

$K$ for disorder strength $W_{K}$ that maximizes the intersample variance $V_{S}$, e.g. $W_{K}=2.7$ for $K=16$. The finite size analysis assures that in the thermodynamic limit $K \rightarrow \infty: W_{K} \rightarrow W_{C}=3.5(1)$ (as discussed in Section II).

As the system size increases the statistics on the ergodic (MBL) side of crossover tend towards GOE (Poisson) limit, the width of the crossover diminishes. The critical level statistics which we conjecture to be relevant exactly at the MBL transition in large system size limit is presented in Fig. 11. The obtained wSRPM contains SRPMs with long-range interactions $h>1$ (non-zero weights $c_{i}$ with $i>30$ ) together with dominating contribution of models with local interactions and $\beta<1$. Large number of contributing SRPMs allows to accurately reproduce the $P\left(r_{S}\right)$ distribution (Fig. 12). Moreover, it is vital to faithfully reproduce the number variance. The values of spectral compressibility $\chi$ defined by the linear large $L$ behavior of the number variance $\Sigma^{2}(L) \propto \chi L$ together with the average gap ratios $\bar{r}$ are shown in Tab. III. 
This quantities are in good agreement with the predictions of the wSRPM $\bar{r}_{w S R P M}$ and $\chi_{w S R P M}$. The data suggest that the remaining small deviation in the spectral compressibility $\chi$ is probably a finite size effect.

Level spacing distribution $P_{A}(s)$ in the Anderson transition [20] combines level repulsion at small $s$ characteristic for GOE and an exponential tail of Poisson level statistics, the critical statistics shown in Fig. 11 also possess the two features. However, the large inter-sample randomness encoded in broad $P\left(r_{S}\right)$ distribution is a crucial property of the critical level statistics in MBL transition, whereas it does not play a role in the Anderson transition in which the $P_{A}\left(r_{S}\right)$ distribution has a Gaussian shape the same width as in the GOE and Poisson limits.

\section{UNIVERSALITY}

The wSRPM model has so far been used to describe level statistics in the standard model of MBL - the XXZ spin chain (2). It has already been noted in [58] that there are differences in level statistics across the MBL transition in systems of hard-core bosons and fermions. In this section we demonstrate that wSRPM can faithfully reproduce level statistics in ergodic to MBL crossover in a disordered Bose-Hubbard model [47] as well as in disordered Fermi-Hubbard model [42].

The system of disordered bosons is described by the Bose-Hubbard Hamiltonian

$$
H_{B}=-J \sum_{\langle i, j\rangle} \hat{a}_{i}^{\dagger} \hat{a}_{j}+\frac{U}{2} \sum_{i} \hat{n}_{i}\left(\hat{n}_{i}-1\right)+\sum_{i} \mu_{i} \hat{n}_{i}
$$

where $a_{i}^{\dagger}, a_{i}$ are bosonic creation and annihilation operators respectively, the tunneling amplitude $J=1$ sets the energy scale, $U$ is interaction strength and the chemical potential $\mu_{i}$ is distributed uniformly in an interval $[-W ; W]$. This model have been shown to be MBL [47] above a critical disorder strength $W_{B}$ which depends on the interaction strength $U$. The Hamiltonian for disordered fermions reads

$$
H_{F 0}=-J \sum_{i, \sigma=\uparrow, \downarrow}\left(\hat{c}_{i \sigma}^{\dagger} \hat{c}_{i+1 \sigma}+h . c\right)+U \sum_{i} n_{i \uparrow} n_{i \downarrow}+\sum_{i} \mu_{i} \hat{n}_{i}
$$

where $c_{i}^{\dagger} c_{i}$ are fermionic creation and annihilation operators respectively; $J=1$ and $U$ are tunneling and interaction amplitudes and $\mu_{i} \in[-W ; W]$ is uncorrelated disorder. To avoid integrability in the absence of disorder it is sufficient [42] to add the next-to-nearest neighbor tunneling terms

$$
H_{1}=-J^{\prime} \sum_{i, \sigma}\left(\hat{c}_{i \sigma}^{\dagger} \hat{c}_{i+2 \sigma}+h . c\right)
$$

and an additional symmetry breaking term

$$
H_{S B}=h_{B}\left(n_{i \uparrow}-n_{i \downarrow}\right)+\mu_{B}\left(n_{L \uparrow}+n_{L \downarrow}\right) .
$$

Transition between GOE and PS statistics for the system with the full Hamiltonian

$$
H_{F}=H_{F 0}+H_{1}+H_{B S}
$$

has been observed in [42].

Level statistics as a function of disorder strength in the bosonic (16) and fermionic (20) models together with wSRPM fits are presented in Fig. 13. Similarly as in the case of XXZ spin chain, the level spacing distributions $P(s)$ are characterized by exponential tails (which are also bending upwards for large $s$ ), the number variance $\Sigma^{2}(L)$ is growing linearly at large $L$ similarly as in the case of disordered XXZ chain. It seems that these are universal features of level statistics in ergodic to MBL crossover in models with short-range interactions. Such systems host an extensive number of LIOMs. Presumably, the observed common features of level statistics across the MBL crossover are associated with the way in which the LIOMs get delocalized as the disorder strength decreases.

Predictions of wSRPM reproducing appropriate $P\left(r_{S}\right)$ distributions for both systems are denoted by solid lines in Fig. 13. In the case of the fermionic system, the method of determining the wSRPM model was exactly the same as for the XXZ spin chain, whereas for bosons we have also included SRPM with $\beta=1$ and $h=25$ (it has $\bar{r}_{\beta=1}^{h=25}=0.5302$ which can be compared with $\left.r_{\beta=1}^{h=5}=0.5262\right)$. For the smallest disorder strengths $W=8.5(W=4.5)$ for bosons (fermions), a single SRPM with $h=12(h=13)$ was fitted. The bulks of level spacing distributions as well as the tails are reproduced reasonably accurately by the wSRPM across the whole MBL crossover. The wSRPM prediction for the number variance $\Sigma^{2}(L)$ is compatible with data for disordered Fermi-Hubbard model. However, the number variance is significantly $20-30 \%$ overestimated for the disordered Bose-Hubbard model at $W=10$ and $W=12$. Inspecting closely the $P\left(r_{S}\right)$ distributions in Fig. 14 we can clearly see abundance of disordered realizations with $r_{S} \gtrsim 0.57$ for $W=10,12$ such that $P\left(r_{S}\right)$ is above the GOE distribution. Precisely this abundance lead us to consider also the $\beta=1$ and $h=25$ SRPM in the fits for bosonic system. While it diminishes the deviation of the number variance, it is clearly insufficient to yield correct spectral compressibility. This demonstrates model specific long-range correlations between eigenvalues that cannot be grasped straightforwardly by wSRPM.

Apart from the model specific details, the exponential tails of level spacing distributions and the finite spectral compressibility that appear already deeply in the metallic phase were observed for the XXZ spin chain as well as in the bosonic and fermionic systems. The wSRPM model is able to grasp all of those features which provides an argument in favor of its generality. Moreover, distinct numbers of rare events occur in various systems during MBL transition which reveals itself in dissimilar correspondences between the bulk of level spacing distribution and its tail as well as the number variance. In 

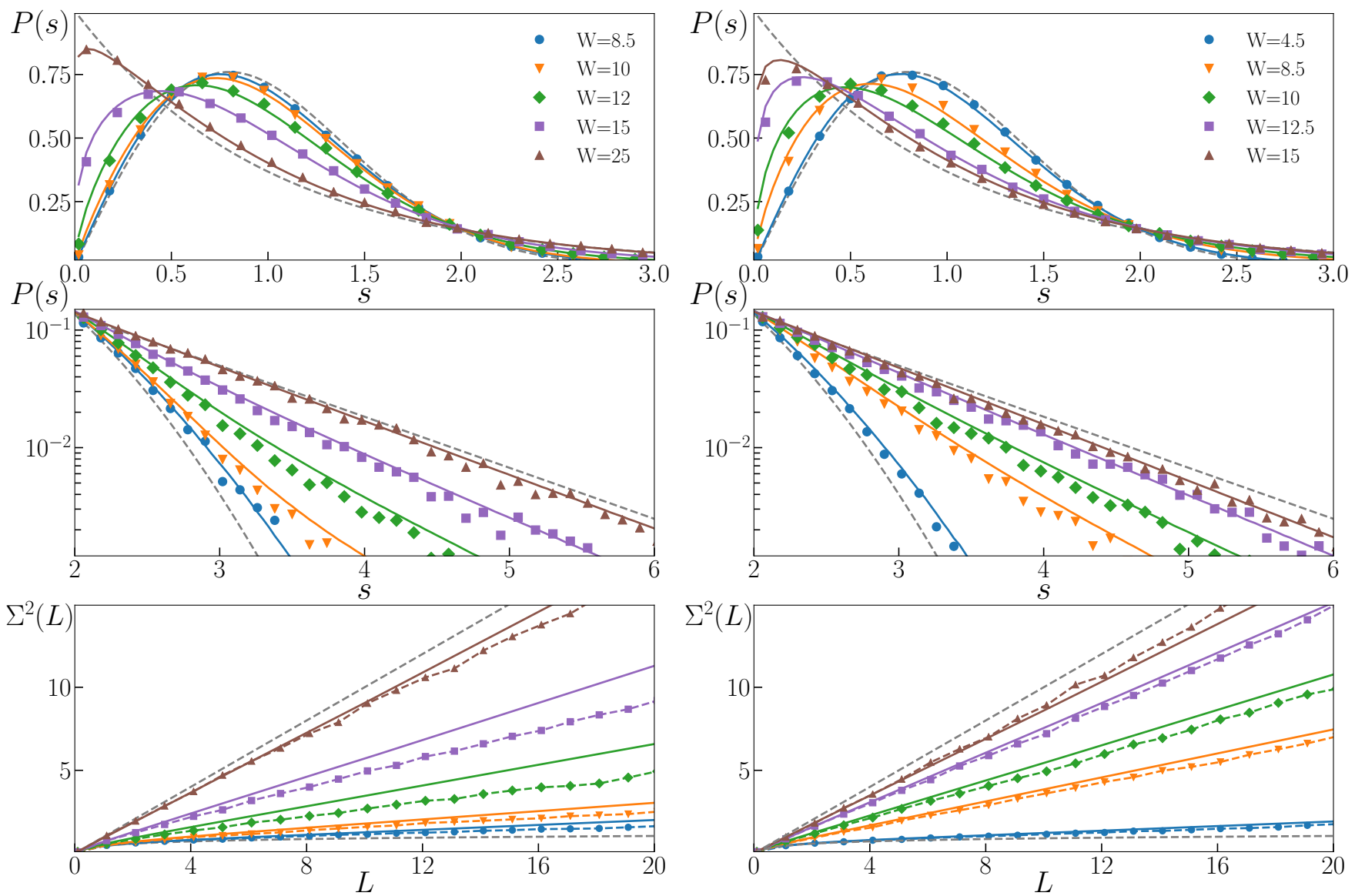

Figure 13. Left: Level spacing distribution $P(s)$ (in lin-lin scale and in lin-log scale) together with number variance $\Sigma^{2}(L)$ during MBL transition in disordered Bose-Hubbard model (16). Results for $N=12$ bosons on $K=8$ lattice sites, interaction amplitude $U=1$ are denoted by markers, solid lines show wSRPM model fits. Right: Level statistics for the Fermi-Hubbard model $H_{F}$. Results for $N_{\uparrow}=3=N_{\downarrow}$ fermions on $K=12$ lattice sites with interaction strength $U=2$ and $\mu_{B}=h_{B}=0.1$, $J^{\prime}=0.5$ are denoted by markers, solid lines correspond to wSRPM model predictions.

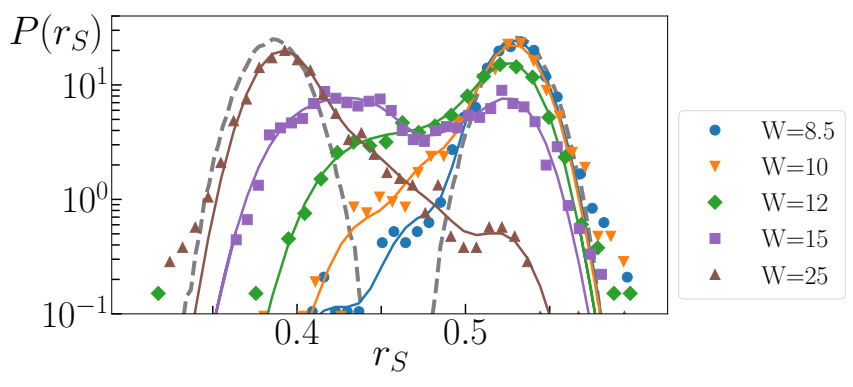

Figure 14. The fit of $P\left(r_{S}\right)$ distributions. Data for bosonic system (16) are denoted with markers, solid lines show wSRPM fits.

general, different systems are characterized by different inter-sample randomness during the MBL transition compare for instance the shape of $P\left(r_{S}\right)$ distributions displayed in Fig. 14 with data for the XXZ spin chain in Fig. 5. This demonstrates that an accurate model of level statistics must be flexible enough to reproduce var- ious types of inter-sample randomness across the MBL crossover - this necessitates an introduction of a weighted model like wSRPM.

\section{LONG-RANGE SPECTRAL CORRELATIONS}

The number variance $\Sigma^{2}(L)$ at large $L$ reflects correlations between energy levels which lie far apart in the spectrum of a system. Such long-range correlations between eigenvalues are strong in the GOE ensemble, resulting in the so called spectral rigidity which is apparent in the asymptotic behavior of the number variance $\Sigma_{G O E}^{2}(L) \rightarrow \log (L)$ at $L \gg 1$. The spectral rigidity of GOE is associated with the fact that the logarithmic interactions act between all pairs of eigenvalues in the JPDF for GOE (7). And it is the spectral rigidity of $\beta$-Gaussian model which causes the large discrepancy between its prediction and the number variance for XXZ spin chain in Fig. 3. On the other hand, the SRPMs de- 
scribe interactions only among a finite number $h$ of neighboring eigenvalues which results in the spectral compressibility of those models $\Sigma_{G O E}^{2}(L) \rightarrow \chi L$ at $L \gg 1$, with $0<\chi<1$. The resulting spectral compressibility of the wSRPM model allows to grasp the linear behavior of number variance in the MBL crossover. The similar behavior can be also obtained with wPLRBM as presented in the preceding section.

To be able to compare statistical properties of eigenvalues from different parts of spectra of various systems, one has to perform the unfolding of energy levels [11] - the procedure of setting mean level spacing to unity. Unfortunately, the number variance $\Sigma^{2}(L)$ is very sensitive to details of the unfolding [59] which has already been a source of discrepancies in descriptions of level statistics in the MBL transition [35, 36]. Consider a set of eigenvalues $\left\{E_{i}\right\}$ ordered in an ascending manner. During the unfolding, a level staircase function $\sigma(E)=\sum_{i} \Theta\left(E-E_{i}\right)$ is separated into smooth and fluctuating parts $\sigma(E)=\bar{\sigma}(E)+\delta \sigma(E)$ and the eigenvalues are mapped via

$$
E_{i} \rightarrow \epsilon_{i}=\bar{\sigma}\left(E_{i}\right)
$$

The difficulty of unfolding lies in an ambiguity of the definition of the smooth part $\bar{\sigma}(E)$ of the staircase function. The most common way is to fit the staircase function $\sigma(E)$ for each disorder realization with a polynomial of a small degree which determines the smooth part $\bar{\sigma}(E)$.

In our case, a set of $n=400$ consecutive eigenvalues is gathered and the resulting level staircase is fitted with a straight line which defines the smooth part $\bar{\sigma}(E)$ used in the unfolding of energy levels. For each disorder realization 7 non-overlapping sets of $n=400$ eigenvalues from the middle of spectrum are taken - effectively employing $\approx 20 \%$ of the spectrum to the analysis as the matrix size for $K=16$ is equal to 12870 . The finite size $n$ of the set of eigenvalues introduces a correction $-a_{2} L^{2} / n$ to the number variance [60]. Carrying out the unfolding with $n=50,100,200,400,800$ we verify that it is indeed the case. We perform a quadratic fit to $\Sigma^{2}(L)$ in the interval $L \in[10,70]$ and obtain the coefficient $a_{2}$ which is weakly dependent on the chosen $L$ interval. Therefore, in order to eliminate the quadratic correction and thus to get rid of the finite $n$ effects we subtract the $-a_{2} L^{2} / n$ term from the number variance data. Let us note that unfolding with finite number $n$ of energy levels can have two consequences. For eigenvalues which are strongly correlated at large distances (e.g. GOE), it destroys level correlations at approximately $n$ level spacings meaning that at this ranges the eigenvalues become uncorrelated. Hence, the number variance becomes overestimated at $L \approx n$. The converse is true for uncorrelated energy levels - unfolding based on $n$ energy levels introduces correlations between them at a certain scale - and the number variance is underestimated. We have checked that our unfolding procedure (together with the $-a_{2} L^{2} / n$ term subtraction) allows us to get correct number variances in the two limiting cases of GOE and PS statistics up to $L \approx 100$.

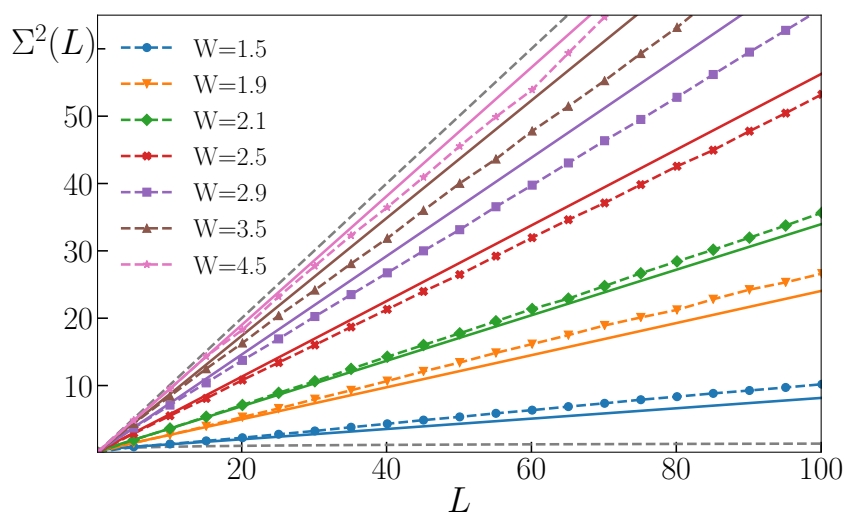

Figure 15. Long-range spectral correlations visible in large $L$ behavior of the number variance $\Sigma^{2}(L)$ for XXZ spin chain (2) during the MBL transition. The dashed lines denote predictions of the wSRPMs with parameters from Tab. I.

The number variances for the XXZ spin chain (2) at various disorder strengths $W$ together with the wSRPM results from Sec. V A are presented in Fig. 15. Nearly perfect agreement between the XXZ spin chain data and the predictions of wSRPM visible in Fig. 6 for $L \in[0,20]$ is lost. Small deviations from the linear behavior of the number variance predicted by wSRPM appear at larger scales which was also indicated by the slight discrepancies between spectral compressibility $\chi$ of the data and the prediction of wSRPM. There are two distinct regimes. For metallic systems with disorder strengths $W \lesssim 2.1$ the number variance obtained from the wSRPM is smaller than the result for XXZ spin chain. This indicates that there exists a regime (for $L \gtrsim 20$ ) where the number variance grows faster than linearly which was interpreted in [36] as a signature of anomalous Thouless energy in the system [61]. We indicate below that this behavior of the number variance for large $L$ has to be examined with an uttermost caution. The second regime arises as the disorder strength increases above $W \approx 2.5$. Then, the number variance predicted by wSRPM slightly overestimates the number variance for the XXZ spin chain. As we have shown in Fig. 9. this effect diminishes as one changes the system size from $K=14$ through $K=16$ to $K=18$ and thus it is likely a finite size effect. However, we cannot completely exclude the possibility that there are some remaining long-range correlations between eigenvalues in the system which are not grasped within the wSRPM.

The simple form of JPDF of wSRPM allows us to get further insight into long-range spectral correlations of system in the MBL crossover. The situation in which wSRPM accurately reproduces level statistics up to 1020 level spacings but underestimates the number variance for $L \gtrsim 20$ is at first sight paradoxical. The wSRPM incorporates interactions between energy levels only at a finite range $h=\max \left\{h_{i}\right\}$. In addition, the weaker the correlation between eigenvalues separated by a given distance the bigger is the number variance at $L$ correspond- 


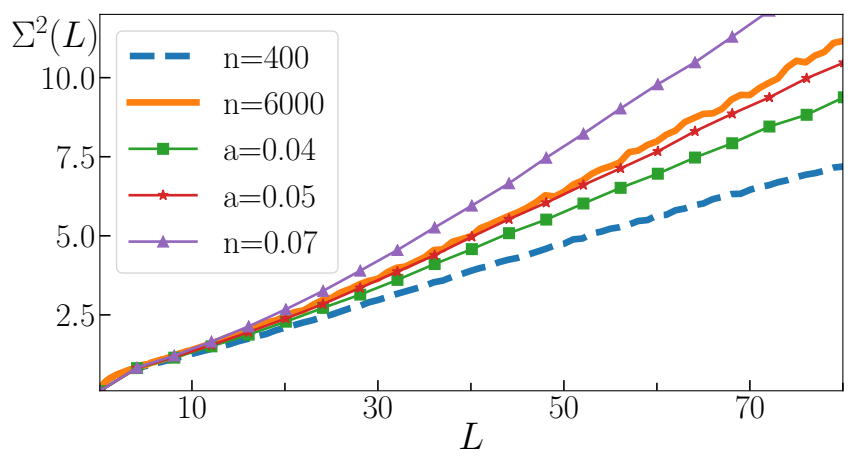

Figure 16. The number variance $\Sigma^{2}(L)$ for XXZ spin chain (2) of size $K=18$ for $W=1.5$. The dashed line show result for local linear unfolding with $n=400$, the solid line for unfolding based on mean density of states [36] with $n=6000$. The number variance $\Sigma^{2}(L)$ obtained after introducing of fluctuations of density of eigenvalues with parameter $a$ are denoted with lines with markers.

ing to this distance. How it is, therefore, possible that wSRPM grasps faithfully the level statistics at the local scale but predicts stronger correlations at larger scales as compared to the data for the XXZ spin chain while at the same time it does not assume presence of any interactions between energy levels beyond the range $h$ ? It turns out that fluctuations of density of eigenvalues on scales of tens level spacings increase the number variance at large $L$. This is precisely the moment in which the unfolding enters the scene as it is the way in which the $\bar{\sigma}(E)$ is defined which determines whether the density fluctuations are incorporated into $\bar{\sigma}(E)$ resulting in number variance $\Sigma^{2}(L)$ growing linearly with $L$ (or, conversely, they are not incorporated and then $\Sigma^{2}(L)$ increases faster than linearly for large $L$ ).

The work [36] reports that the number variance grows according to a power-law $\Sigma^{2}(L) \propto L^{\gamma}$ with $\gamma>1$ for large $L$ for the XXZ spin chain (2) deep in the metallic regime where the exponent $\gamma$ acquires values up to $\gamma \approx 1.4$. The number variance obtained by us for $W=1.9$ has clearly some region in which it increases faster than linearly, but such a power-law growth is not observed by us. This discrepancy has its root in the unfolding. Unfolding employed in [36] relies on assumption that the shape of mean density of states obtained for the system at given disorder strength can be used (after appropriate linear transformations) to unfold large portions of spectrum of the system taking $n \approx 6000$ consecutive energy levels for $K=18$. The fluctuations of density of eigenvalues on the scales of tens or hundreds of eigenvalues which are different for different disorder realizations are not incorporated in the $\bar{\sigma}(E)$ as it is determined by the mean density of states in which such fluctuations are averaged out.

Fig. 16 compares the number variances obtained after the local linear unfolding with $n=400$ consecutive eigenvalues and after the unfolding of [36]. The results agree up to $L \approx 15$. In order to show that the difference between the results stems from the density fluctuations we introduce a particular density modulation to the data from the local linear unfolding. Namely, the unfolding is modified so that the eigenvalues are mapped via

$$
E_{i} \rightarrow \epsilon_{i}=\bar{\sigma}\left(E_{i}\right)+a\left(E_{i}-E_{C}\right)^{2},
$$

where $E_{C}$ lies in the middle of the energy interval which is unfolded. The $a\left(E_{i}-E_{C}\right)^{2}$ term mimics the density fluctuations which were not incorporated into $\bar{\sigma}(E)$, for $a=0$ (22) reduces to the local linear unfolding (21). Such a density modulation does not alter $P(s)$ at all, however, it modifies the number variance exactly in the manner which allows us to reproduce the result of [36] and showing that the density fluctuations are the mechanism which causes the power-law growth of the number variance.

In conclusion, the behavior of the number variance $\Sigma^{2}(L)$ suggests that long-range spectral correlations might be present in the level statistics of XXZ spin chain during MBL transition. This feature of MBL transition lies beyond the scope of wSRPM, however, as we demonstrated by examining bosonic and fermionic systems it is model dependent. It is not clear whether the unfolding employed in [36] is justified. As we have indicated, it does not take into account variations of density of eigenvalues at scales of tens and hundreds of level spacings for a given disorder realization. Let us emphasize once again that the situation of localization of an interacting system differs starkly from the usual RMT where a random matrix depends on number of random entries which scales as square of its size whereas the number of random entries in the Hamiltonian of the XXZ spin chain scales only as logarithm of the size of the Hilbert space of the system. Therefore one may expect that while the density fluctuations average out for RMT and using Wigner's semi-circle to unfold GOE is a good idea it may not be the case for the many body quantum systems which undergo MBL transition.

\section{CONCLUSIONS AND BEYOND}

Analyzing the gap ratios of systems in the crossover between ergodic and many-body localized regimes we have shown that a complete information about interand intra-sample randomness can be obtained from the $r_{n}$ variables. Distribution $P\left(r_{S}\right)$ of the sample averaged gap ratio $r_{S}$ provides a suggestive illustration of Griffiths regions in the case of random disorder while it shows absence of such rare events in systems with quasiperiodic disorder. The proposed inter- and intra-sample variances $V_{S}$ and $V_{I}$ provide a straightforward method to quantify inter- and intra-sample randomness. While our analysis provides further insights into role played by Griffiths regions in MBL transition, it is conceptually and computationally (involves only eigenvalues) simple and therefore can be straightforwardly employed in studies of other sys- 
tems where a transition between integrable and RMT-like regimes occurs.

Examining the bulk and the tail of level spacing distribution together with the number variance we have demonstrated that the proposed models of spectral statistics in MBL crossover [35, 36, 38, 39] grasp level statistics accurately only at the level of few of level spacings. To reproduce broad distributions of the sample averaged gap ratio $r_{S}$ in the MBL transition we have introducted the wSRPM that is a statistical mixture of the well known family of short-range plasma models. The wSRPM describes faithfully the flow of level statistics in the whole ergodic to MBL crossover. According to wSRPM the correlations between eigenvalues are present only at a finite range $h$. In the ergodic phase the range $h$ diverges resulting in GOE statistics and as the system flows towards MBL phase the range of correlations diminishes. At a certain point the interactions become local $(h=1)$, finally in the vicinity of MBL phase the level repulsion vanishes $(\beta \rightarrow 0)$ resulting in the Poisson statistics. The wSRPM grasps universal features of level statistics across MBL transition in a variety of spin, bosonic and fermionic systems with interactions and random disorder. The assumption that there are no correlations between eigenvalues at ranges larger than $h$ predicts the finite spectral compressibility $\chi$ in the transition. The latter seems to be approximately true for the studied systems albeit small deviations from the linear behavior of the number variance have been noticed. This may be either an artifact of the unfolding procedure or could also stem from weak long-range interactions between energy levels which are model and system size dependent.

We also considered a weighted ensemble of power law banded random matrices - see Appendix II. An appropriate mixture of PLBRM (again necessitated by a broad distribution of gap ratio in physical samples) seems to be at least competitive with wSRPM leading to small deviations of the fitted model from the data for XXZ spin chain. Both approaches have their advantages. While for SRPMs the eigenvalues may be generated by brute force Monte Carlo integration of the JPDF, a softer semianalytic approach, working at certain range of eigenvalues interaction, $h$, is possible following the path shown by Bogomolny and coworkers [55] as shown in Appendix I. It provides expressions for the level spacing distribution $P(s)$ and, more importantly, gives analytical formulas for asymptotic behavior of the number variance $\Sigma^{2}(L)$ as well as for the tails of $P(s)$. Moreover, the wSRPM gives a concrete microscopic description of correlations between eigenvalues across the whole MBL crossover and allows us to speculate how the level statistics evolve in the limit of large system sizes.

On the other hand that approach provides us with no clue on the eigenvectors behavior. On the contrary, PLBRM model provides access to both eigenvalues and eigenvectors by a direct (although costly) diagonalization of a large number of matrices from the ensemble. The drawback of this approach is that there are no an- alytical results for this model at finite $N$ or $\mu \neq 1$ so a clear picture of correlations between eigenvalues is not available.

Finally, it is also interesting to note that the MBL transition for the quasiperiodic disorder case cannot be described by the proposed weighted ensembles. It supports the claim of [33] that the transitions for RD and QPD are of different universality classes. The ensemble that reproduces level statistics for QPD in MBL transition is yet to be identified.

\section{ACKNOWLEDGMENTS}

We acknowledge fruitful and enlightening discussions with D. Delande as well as exchanges on unfolding procedures with A. M. Garcia-Garcia and M. Sieber. This work was performed with the support of EU via Horizon2020 FET project QUIC (nr. 641122). Numerical results were obtained with the help of PL-Grid Infrastructure. We acknowledge support of the National Science Centre (PL) via project No.2015/19/B/ST2/01028 (P.S.), No.2018/28/T/ST2/00401 (Etiuda scholarship - P.S.) and the QuantERA programme No. 2017/25/Z/ST2/03029 (J.Z.).

\section{APPENDIX: ANALYTICAL EXPRESSIONS FOR SHORT-RANGE PLASMA MODEL}

The following quantities are needed in order to construct wSRPM: level spacing distributions $P_{h}^{\beta}(s)$, number variances $\Sigma_{\beta, h}^{2}(L)$, the sample gap ratio distribution $P_{h}^{\beta}\left(r_{S}\right)$ of the individual SRPMs used in the wSRPM. Semi-analytic expression for most of those quantities have been given in Ref. [55]. We comment here on how they can be used in the context of wSRPM.

Let us start with $h=1$. The distribution of level spacing between $n$-th neighboring eigenvalues reads

$$
P_{h=1}^{\beta}(n, s)=\frac{(\beta+1)^{(n+1)(\beta+1)}}{\Gamma((\beta+1)(n+1))} s^{\beta+n(\beta+1)} \mathrm{e}^{-(\beta+1) s},
$$

where $\beta \in[0,1]$, for $n=0$ this distribution reduces to the usual level spacing distributions. For $n \geq 0$, the formula (A.1) can be used to obtain the number variance according to the following general expression

$$
\Sigma^{2}(L)=L-2 \int_{0}^{L} \mathrm{~d} s(L-s)\left(1-\sum_{n=0}^{\infty} P(n, s)\right)
$$

From our Monte Carlo evaluation of JPDF (8) we know that the $P_{h}^{\beta}\left(r_{S}\right)$ distributions (for range $h$ and $\beta$ relevant in applications of our wSRPM) are Gaussian functions, localized around certain values $\bar{r}_{h}^{\beta}$ with standard deviation $\sigma \approx 0.01557$ which changes only very slightly for various $h$ and $\beta$. Therefore, instead of deriving the full 
$P_{h=1}^{\beta}\left(r_{S}\right)$ distribution, we simply use the distribution of gap ratio to calculate $\bar{r}_{h=1}^{\beta}$ and use the Gaussian approximation for the full distribution $P_{h=1}^{\beta}\left(r_{S}\right)$. The gap ratio distribution $P_{h=1}^{\beta}(r)$ reads [62]

$$
P_{h=1}^{\beta}(r)=\frac{\Gamma(2 \beta+2) \Gamma^{2}(\beta+2)}{(\beta+1)^{2} \Gamma^{4}(\beta+1)} \frac{2 r^{\beta}}{(1+r)^{2 \beta+2}},
$$

from which we get $\bar{r}_{h=1}^{\beta}$ as the first moment of $P_{h=1}^{\beta}(r)$ distribution.

For $h>1$ the only interesting case for us is $\beta=1$. Then, the level spacing distribution is given by (9). In order to determine the polynomial $W(s)$ as well as the distributions $P_{h}^{\beta}(n, s)$ for $h>1$ and $n>0$ one needs to solve the integral equation[55]

$$
\begin{array}{r}
\int_{0}^{\infty} \mathrm{d} \xi_{h} \mathrm{e}^{-\xi_{h}} \xi_{h}\left(\xi_{h}+\xi_{h-1}\right) \ldots\left(\xi_{h}+\ldots+\xi_{1}\right) \psi_{j}\left(\xi_{2}, \ldots, \xi_{h}\right) \\
=\lambda_{j} \psi_{j}\left(\xi_{1}, \ldots, \xi_{h-1}\right),
\end{array}
$$

for function $\psi_{j}\left(\xi_{1}, \ldots, \xi_{k-1}\right)$. The equation can be solved by a polynomial ansatz

$$
\begin{aligned}
& \psi_{j}\left(\xi_{1}, \ldots, \xi_{h-1}\right)= \\
& \sum_{i_{1}=0}^{1} \sum_{i_{2}=0}^{3} \ldots \sum_{i_{h-1}=0}^{(h-1) h / 2} a_{i_{1} i_{2} \ldots i_{h-1}} \xi_{1}^{i_{1}} \xi_{2}^{i_{2}} \ldots \xi_{h-1}^{i_{h-1}}
\end{aligned}
$$

which reduces (A.4) to an eigenproblem for a matrix of dimension $D_{h}=\prod_{k=1}^{h-1} i_{k}$. Solving the eigenproblem, the eigenfunctions $\psi_{j}\left(\xi_{1}, \ldots, \xi_{h-1}\right)$ can be used to find $P_{h}^{\beta}(n, s)$ for $h>1$ and $n \geq 0$ as well as $P_{h}^{\beta}(r)$ which is then used to determine $P_{h}^{\beta}\left(r_{S}\right)$ as a Gaussian function with standard deviation $\sigma=0.01557$ centered around $\bar{r}_{h}^{\beta}$. The level spacing distribution is determined as $P_{h}^{\beta}(s)=P_{h}^{\beta}(n=0, s)$, whereas $P_{h}^{\beta}(n=0, s)$ for $5>n \geq 0$ are used to find the number variance $\Sigma_{\beta, h}^{2}(L)$ for $L<3$. For larger values $L \geq 3$ the asymptotic form (10) of the number variance is used.

Unfortunately, the dimension $D_{h}$ grows exponentially with $h$ which makes the semi-analytic approach feasible only for $h<6$ which incidentally covers all the SRPMs used in (14). The coefficients which determine $P_{h}^{\beta}(n, s)$ are gathered in Tab. IV, V, VI,VII. The $P_{h}^{\beta=1}\left(r_{S}\right)$ distributions are approximated as Gaussian distributions with standard deviation $\sigma=0.01557$ centered around the semi-analytically obtained values of $\bar{r}_{h}^{\beta=1}$ which are $0.5155,0.5206,0.5231,0.5246$ for $h=2,3,4,5$ respectively.

\section{APPENDIX: WEIGHTED POWER-LAW RANDOM BANDED MATRICES}

The wSRPM describes faithfully level statistics in MBL transition. However, it provides no information on properties of eigenstates. One particularly interesting property is multifractality of matrix elements of local
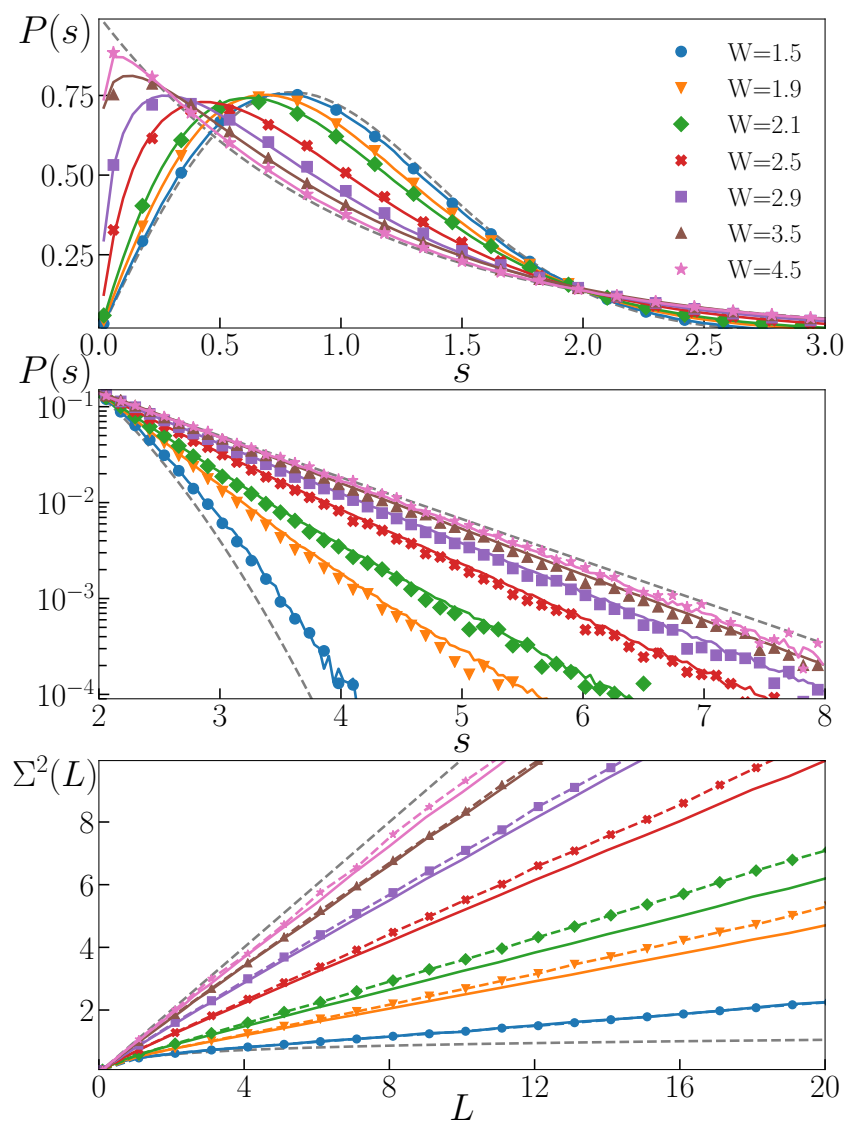

Figure 17. Distributions $P\left(r_{S}\right)$ across the MBL transition (denoted by markers) with fits from the weighted PLRBM model.

operators $[63,64]$ in such states. Therefore, an identification of a random matrix model which could provide some information about eigenvectors in MBL transition can be productive.

In this Appendix we examine an ensemble of powerlaw random banded matrices (PLRBM) [24, 65] which is the ensemble of $N \times N$ symmetric real matrices with matrix elements $H_{i j}$ being independent random Gaussian variables with

$\left\langle H_{i j}\right\rangle=0$ and $\left\langle H_{i j}^{2}\right\rangle=\left(1+\delta_{i j}\right)\left(1+(|i-j| / B)^{2 \mu}\right)^{-1}$

This ensemble interpolates between GOE statistics for $B \gg 1, \mu<1$ and PS statistics which arises for $\mu>1$ in $N \rightarrow \infty$ limit. In the special case of $\mu=1$ and large $B$ the model can be solved by a mapping onto an effective $\sigma$ model [66]. Numerical calculations of level statistics of the PLRBM model at the critical line $\mu=1$ were carried out in $[60,67]$.

We consider PLRBM of size $N=1000$, accumulating 10000 matrices for each set of parameters $(\mu, B)$. Let us note that the exact values of the $(\mu, B)$ coefficients are strongly dependent on size $N$ of matrix from PLRBM. With growing $N$ a flow of level statistics in 


\begin{tabular}{l|lllll}
\hline & $n=0$ & $n=1$ & $n=2$ & $n=3$ & $n=4$ \\
\hline$w_{1}$ & 2.4773 & 2.50542 & 0.325782 & 0.0160653 & $1.9203 \cdot 10^{-4}$ \\
$w_{2}$ & 6.06811 & 3.0685 & 0.243833 & $6.51294 \cdot 10^{-3}$ & $8.2295 \cdot 10^{-5}$ \\
$w_{3}$ & 3.71594 & 0.751625 & $4.0723 \cdot 10^{-2}$ & $6.0483 \cdot 10^{-4}$ & $8.2297 \cdot 10^{-6}$ \\
\hline
\end{tabular}

Table IV. Coefficients, $h=2, P(n, s)=\exp (-3 s) s^{3 n} \sum_{j} w_{j} s^{j}$

\begin{tabular}{c|cllll}
\hline & $n=0$ & $n=1$ & $n=2$ & $n=3$ & $n=4$ \\
\hline$w_{1}$ & 2.13422 & 1.13398 & 0.14101 & $3.03041 \cdot 10^{-3}$ & $1.7921 \cdot 10^{-5}$ \\
$w_{2}$ & 7.81963 & 3.63343 & 0.29749 & $4.48832 \cdot 10^{-3}$ & $2.0107 \cdot 10^{-5}$ \\
$w_{3}$ & 11.6945 & 5.01886 & 0.26416 & $2.81378 \cdot 10^{-3}$ & $9.7956 \cdot 10^{-6}$ \\
$w_{4}$ & 9.07429 & 3.71122 & 0.12338 & $9.58731 \cdot 10^{-4}$ & $2.6381 \cdot 10^{-6}$ \\
$w_{5}$ & 3.68554 & 1.50368 & $3.2011 \cdot 10^{-2}$ & $1.87245 \cdot 10^{-4}$ & $4.1257 \cdot 10^{-7}$ \\
$w_{6}$ & 0.62587 & 0.310017 & $4.455 \cdot 10^{-3}$ & $2.00989 \cdot 10^{-5}$ & $3.5835 \cdot 10^{-8}$ \\
$w_{7}$ & 0 & $2.59412 \cdot 10^{-2}$ & $2.6414 \cdot 10^{-4}$ & $9.37094 \cdot 10^{-7}$ & $1.3637 \cdot 10^{-9}$ \\
\hline
\end{tabular}

Table V. Coefficients, $h=3, P(n, s)=\exp (-4 s) s^{4 n-1+\delta_{0, n}} \sum_{j} w_{j} s^{j}$, where $\delta_{i, j}$ denotes the Kronecker delta.

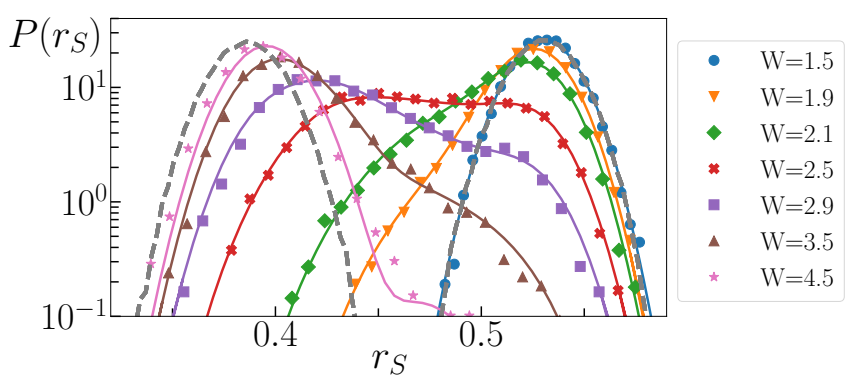

Figure 18. Level statistics of wPLRBM model fitted to data for XXZ spin chain (2) with appropriate $r_{S}$ filtering - details in Tab. III.

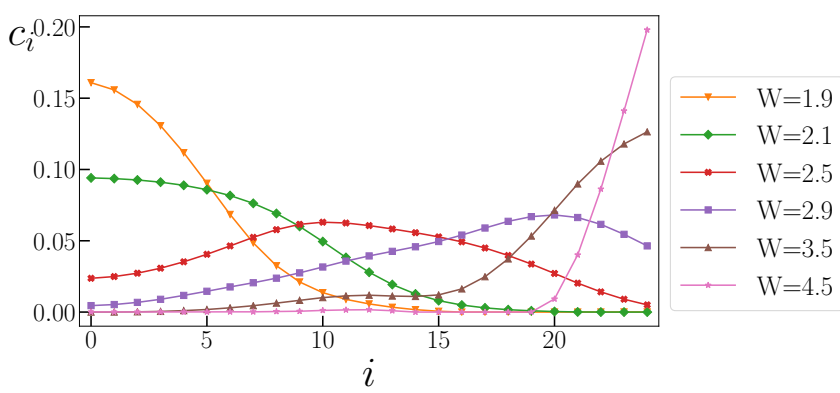

Figure 19. Coefficients $c_{i}$ for the wPLRBM model for XXZ spin chain across the ergodic MBL crossover.

this model occurs - points $(\mu, B)$ with $\mu<1$ correspond to statistics closer and closer to GOE and analogously - for $\mu>1$ statistics flow towards PS. Calculating the $P\left(r_{S}\right)$ distribution for PLRBM model we have verified that $P\left(r_{S}\right)$ remains Gaussian in large region of parameter space $(\mu, B)$. Moreover, there exist a region of parameters for which the level spacing distributions $P_{B}^{\mu}(s)$ decay exponentially and the number variance $\Sigma_{\mu, B}^{2}(L)$ is asymptotically linear. Therefore, a similar extension as in the case of SRPM can be proposed in which the intersample randomness encoded in the $P\left(r_{S}\right)$ distribution is mimicked by considering a mixture of PLRBM with various $\mu_{i}$ and $B_{i}$ to describe the level statistics in given point of MBL transition. More precisely, the selected set of PLRBMs consists of models with $B_{i}=0.35$ and varying $\mu_{i} \in\{0.75+i * 0.025\}_{i=0,1, \ldots 23,22,24,26,28,32,39,49}$. The corresponding weight coefficients are obtained by minimizing $\chi^{2}$ as in (15) with results shown in Fig. 19. The $W=1.5$ was fitted with single PLRBM model with $B=0.35, \mu=0.7$.

Let us note that this model gives very good agreement at the level of tens of level spacings. Tails of the level spacing distributions as well as the number variance obtained from weighted PLRBM model are compatible with the XXZ spin chain data. Certain deviations are visible in the spectral compressibility $\chi$ at larger $L$.

The PLRBM model was introduced as a model for studies of critical properties of Anderson localization. In its direct interpretation the model (A.6) describes a single particle on one dimensional sample with disorder and with long-range hopping - tunneling amplitude decays according to a power-law with distance. Our results show that the PLRBM can be used also in MBL transition provided the weighted mixture of matrices is considered.

Such an ensemble needs to be introduced to mimic the large inter-sample randomness in the MBL crossover, which is a specific feature of localization of an interacting system, whose exponentially large in $L$ Hamiltonian matrix depends only on $L$ random variables.

One way of interpreting this result is that MBL can be thought of as a single particle localization in a 'Fockspace lattice' with complex geometry [68, 69] (reflecting the quantum many-body character of the phenomenon). Another approach is to view wPLRBM as the Hamiltonian of the system at late stages of diagonalization flow [70-72] so that the diagonal entries represent random eigenergies associated with soon-to-be LIOMs and the 


\begin{tabular}{c|llll}
\hline & $n=0$ & $n=1$ & $n=2$ & $n=3$ \\
\hline$w_{1}$ & 1.9869 & 0.810475 & 0.0483296 & $7.29136 \cdot 10^{-4}$ \\
$w_{2}$ & 9.44665 & 3.61582 & 0.190586 & $2.12001 \cdot 10^{-3}$ \\
$w_{3}$ & 20.1982 & 7.54195 & 0.351477 & $2.85649 \cdot 10^{-3}$ \\
$w_{4}$ & 25.6905 & 9.67193 & 0.399671 & $2.35667 \cdot 10^{-3}$ \\
$w_{5}$ & 21.5383 & 8.44298 & 0.310511 & $1.32441 \cdot 10^{-3}$ \\
$w_{6}$ & 12.39 & 5.24733 & 0.172672 & $5.34180 \cdot 10^{-4}$ \\
$w_{7}$ & 4.90293 & 2.35889 & $7.01559 \cdot 10^{-2}$ & $1.58784 \cdot 10^{-4}$ \\
$w_{8}$ & 1.28897 & 0.763259 & $2.0934 \cdot 10^{-2}$ & $3.51389 \cdot 10^{-5}$ \\
$w_{9}$ & 0.204419 & 0.17333 & $4.55485 \cdot 10^{-3}$ & $5.76574 \cdot 10^{-6}$ \\
$w_{10}$ & 0.0148948 & $2.61995 \cdot 10^{-2}$ & $7.06631 \cdot 10^{-4}$ & $6.86517 \cdot 10^{-7}$ \\
$w_{11}$ & 0 & $2.36573 \cdot 10^{-3}$ & $7.44424 \cdot 10^{-5}$ & $5.64602 \cdot 10^{-8}$ \\
$w_{12}$ & 0 & $9.63045 \cdot 10^{-5}$ & $4.79282 \cdot 10^{-6}$ & $2.87566 \cdot 10^{-9}$ \\
$w_{13}$ & 0 & 0 & $1.42564 \cdot 10^{-7}$ & $6.85001 \cdot 10^{-11}$ \\
\hline
\end{tabular}

Table VI. Coefficients, $h=4, P(n, s)=\exp (-5 s) s^{(n+1)(n+4) / 2-2} \sum_{j} w_{j} s^{j}$.

\begin{tabular}{c|llll}
\hline & $n=0$ & $n=1$ & $n=2$ & $n=3$ \\
\hline$w_{1}$ & 1.90607 & 0.6725112 & $2.816938 \cdot 10^{-2}$ & $1.90849 \cdot 10^{-4}$ \\
$w_{2}$ & 11.0655 & 3.757313 & 0.1474950 & $8.91886 \cdot 10^{-4}$ \\
$w_{3}$ & 30.0426 & 10.08246 & 0.37248642 & $2.00465 \cdot 10^{-3}$ \\
$w_{4}$ & 50.783 & 17.24174 & 0.60284124 & $2.87998 \cdot 10^{-3}$ \\
$w_{5}$ & 59.9035 & 21.02683 & 0.70045560 & $2.96413 \cdot 10^{-3}$ \\
$w_{6}$ & 52.2919 & 19.39166 & 0.62020 & $2.32137 \cdot 10^{-3}$ \\
$w_{7}$ & 34.9026 & 13.99334 & 0.43344442 & $1.43410 \cdot 10^{-3}$ \\
$w_{8}$ & 18.1222 & 8.06393 & 0.2443500 & $7.15049 \cdot 10^{-4}$ \\
$w_{9}$ & 7.36415 & 3.75359 & 0.11260234 & $2.92059 \cdot 10^{-4}$ \\
$w_{10}$ & 2.33283 & 1.41789 & $4.27332 \cdot 10^{-2}$ & $9.8650 \cdot 10^{-5}$ \\
$w_{11}$ & 0.567501 & 0.4341132 & $1.339465 \cdot 10^{-2}$ & $2.77077 \cdot 10^{-5}$ \\
$w_{12}$ & 0.102858 & 0.1069601 & $3.464275 \cdot 10^{-3}$ & $6.48527 \cdot 10^{-6}$ \\
$w_{13}$ & $4.05358 \cdot 10^{-5}$ & $2.09130 \cdot 10^{-2}$ & $7.357053 \cdot 10^{-4}$ & $1.26372 \cdot 10^{-6}$ \\
$w_{14}$ & 0 & $3.17047 \cdot 10^{-4}$ & $1.271140 \cdot 10^{-4}$ & $2.04088 \cdot 10^{-7}$ \\
$w_{15}$ & 0 & $3.59091 \cdot 10^{-4}$ & $1.760258 \cdot 10^{-5}$ & $2.70805 \cdot 10^{-8}$ \\
$w_{16}$ & 0 & $2.85564 \cdot 10^{-6}$ & $1.908644 \cdot 10^{-6}$ & $2.908829 \cdot 10^{-9}$ \\
$w_{17}$ & 0 & $1.41936 \cdot 10^{-6}$ & $1.561970 \cdot 10^{-7}$ & $2.44768 \cdot 10^{-10}$ \\
$w_{18}$ & 0 & $3.30973 \cdot 10^{-8}$ & $9.07438 \cdot 10^{-9}$ & $1.62366 \cdot 10^{-11}$ \\
$w_{19}$ & 0 & 0 & $3.33532 \cdot 10^{-10}$ & $5.17973 \cdot 10^{-13}$ \\
$w_{20}$ & 0 & 0 & $5.82950 \cdot 10^{-12}$ & $1.11711 \cdot 10^{-12}$ \\
\hline
\end{tabular}

Table VII. Coefficients, $h=5, P(n, s)=\exp (-6 s) s^{(n+1)(n+4) / 2-2} \sum_{j} w_{j} s^{j}$.

quickly decaying off-diagonal elements account for still present interactions which become weaker and weaker close to the MBL phase.

If the latter is true, then to get the multifractal properties of matrix elements of local operators [63, 64] one has to know transformation between the $\sigma_{i}^{z}$ eigenbasis (in which the Hamiltonian matrix is straightforwardly computed) and the basis in which the Hamiltonian becomes the banded matrix. This would also be the basis in which an interesting relation between the multifractal dimension $D_{1}$ and the spectral compressibility $\chi$ holds. This is beyond the scope of the present paper.
[1] J. Wishart, Biometrika 20A, 32 (1928).

[2] R. A. Janik and M. A. Nowak, Journal of Physics A: Mathematical and General 36, 3629 (2003).

[3] C. W. J. Beenakker, Phys. Rev. Lett. 70, 1155 (1993).

[4] J. J. M. Verbaarschot and I. Zahed, Phys. Rev. Lett. 70, 3852 (1993).

[5] Y. V. Fyodorov and H.-J. Sommers, J. Math. Phys. 38,
1918 (1997), https://doi.org/10.1063/1.531919.

[6] Y. V. Fyodorov and B. A. Khoruzhenko, Phys. Rev. Lett. 83, 65 (1999).

[7] A. Zanella, M. Chiani, and M. Z. Win, IEEE Transactions on Communications 57, 1050 (2009).

[8] J.-P. Bouchaud and M. Potters, Theory of Financial Risks (Cambridge University Press, Cambridge, 2001). 
[9] C. E. Porter, Statistical Theory of Spectra:Fluctuations (Academic, New York, 1965).

[10] M. L. Mehta, Random Matrices (Revised and Enlarged Second Edition) (Elsevier, 1990).

[11] F. Haake, Quantum Signatures of Chaos (Springer, Berlin, 2010).

[12] F. J. Dyson, J. Math. Phys. 3, 140 (1962).

[13] F. J. Dyson, J. Math. Phys. 3, 157 (1962).

[14] F. J. Dyson, J. Math. Phys. 3, 166 (1962).

[15] F. J. Dyson, J. Math. Phys. 13, 90 (1972).

[16] O. Bohigas, M. J. Giannoni, and C. Schmit, Phys. Rev. Lett. 52, 1 (1984).

[17] H. Friedrich and H. Wintgen, Physics Reports 183, 37 (1989).

[18] B. Eckhardt, Physics Reports 163, 205 (1988).

[19] O. Bohigas, S. Tomsovic, and D. Ullmo, Physics Reports 223, 43 (1993).

[20] B. I. Shklovskii, B. Shapiro, B. R. Sears, P. Lambrianides, and H. B. Shore, Phys. Rev. B 47, 11487 (1993).

[21] V. E. Kravtsov, I. V. Lerner, B. L. Altshuler, and A. G. Aronov, Phys. Rev. Lett. 72, 888 (1994).

[22] A. G. Aronov, V. E. Kravtsov, and I. V. Lerner, Phys. Rev. Lett. 74, 1174 (1995).

[23] V. E. Kravtsov and I. V. Lerner, J. Phys. A: Mat. Gen. 28, 3623 (1995).

[24] F. Evers and A. D. Mirlin, Rev. Mod. Phys. 80, 1355 (2008).

[25] D. Basko, I. Aleiner, and B. Altschuler, Ann. Phys. (NY) 321, 1126 (2006).

[26] M. Srednicki, Phys. Rev. E 50, 888 (1994).

[27] M. Schreiber, S. S. Hodgman, P. Bordia, H. P. Lüschen, M. H. Fischer, R. Vosk, E. Altman, U. Schneider, and I. Bloch, Science 349, 842 (2015), http://science.sciencemag.org/content/349/6250/842.full.pdf.

[28] J. Smith, A. Lee, P. Richerme, B. Neyenhuis, P. W. Hess, P. Hauke, M. Heyl, D. A. Huse, and C. Monroe, Nature Physics 12, 907 (2016).

[29] J.-y. Choi, S. Hild, J. Zeiher, P. Schauß, A. RubioAbadal, T. Yefsah, V. Khemani, D. A. Huse, I. Bloch, and C. Gross, Science 352, 1547 (2016), http://science.sciencemag.org/content/352/6293/1547.full.p

[30] V. Oganesyan and D. A. Huse, Phys. Rev. B 75, 155111 (2007).

[31] M. Serbyn, Z. Papić, and D. A. Abanin, Phys. Rev. Lett. 111, 127201 (2013).

[32] V. Ros, M. Mueller, and A. Scardicchio, Nuclear Physics B 891, 420 (2015).

[33] V. Khemani, D. N. Sheng, and D. A. Huse, Phys. Rev. Lett. 119, 075702 (2017).

[34] S.-X. Zhang and H. Yao, ArXiv e-prints (2018), arXiv:1805.05958 [cond-mat.str-el].

[35] M. Serbyn and J. E. Moore, Phys. Rev. B 93, 041424 (2016).

[36] C. L. Bertrand and A. M. García-García, Phys. Rev. B 94, 144201 (2016).

[37] N. Chavda, H. N. Deota, and V. K. B. Kota, Phys. Lett. A 378, 3012 (2014).

[38] P. Shukla, New Journal of Physics 18, 021004 (2016).

[39] W. Buijsman, V. Cheianov, and V. Gritsev, arXiv:1807.05075 [cond-mat, physics:nlin] (2018), arXiv: 1807.05075.

[40] Y. Y. Atas, E. Bogomolny, O. Giraud, and G. Roux, Phys. Rev. Lett. 110, 084101 (2013).
[41] A. Pal and D. A. Huse, Phys. Rev. B 82, 174411 (2010).

[42] R. Mondaini and M. Rigol, Phys. Rev. A 92, 041601 (2015).

[43] D. J. Luitz, N. Laflorencie, and F. Alet, Phys. Rev. B 91, 081103 (2015).

[44] D. J. Luitz, N. Laflorencie, and F. Alet, Phys. Rev. B 93, 060201 (2016).

[45] P. Sierant, D. Delande, and J. Zakrzewski, Phys. Rev. A 95, 021601 (2017).

[46] P. Sierant, D. Delande, and J. Zakrzewski, Acta Phys. Polon. A 132, 1707 (2017).

[47] P. Sierant and J. Zakrzewski, New Journal of Physics 20, 043032 (2018).

[48] J. Janarek, D. Delande, and J. Zakrzewski, Phys. Rev. B 97, 155133 (2018).

[49] D. Wiater and J. Zakrzewski, Phys. Rev. B 98, 094202 (2018).

[50] R. B. Griffiths, Phys. Rev. Lett. 23, 17 (1969).

[51] T. Vojta, J. Low Temp. Phys. 161, 299 (2010).

[52] K. Agarwal, S. Gopalakrishnan, M. Knap, M. Müller, and E. Demler, Phys. Rev. Lett. 114, 160401 (2015).

[53] K. Agarwal, E. Altman, E. Demler, S. Gopalakrishnan, D. A. Huse, and M. Knap, Annalen der Physik, $\mathbf{5 2 9}$ (2016).

[54] K. Kudo and T. Deguchi, Phys. Rev. B 97, 220201 (2018).

[55] Bogomolny, E., Gerland, U., and Schmit, C., Eur. Phys. J. B 19, 121 (2001).

[56] V. E. Kravtsov, I. M. Khaymovich, E. Cuevas, and M. Amini, New Journal of Physics 17, 122002 (2015).

[57] I. Dumitriu and A. Edelman, Journal of Mathematical Physics 43, 5830 (2002).

[58] L. F. Santos and M. Rigol, Phys. Rev. E 81, 036206 (2010).

[59] J. M. G. Gómez, R. A. Molina, A. Relaño, and J. Retamosa, Phys. Rev. E 66, 036209 (2002).

[60] M. L. Ndawana and V. E. Kravtsov, Journal of Physics A: Mathematical and General 36, 3639 (2003).

[61] D. Braun and G. Montambaux, Phys. Rev. B 52, 13903 (1995).

[62] Y. Y. Atas, E. Bogomolny, O. Giraud, P. Vivo, and E. Vivo, Journal of Physics A: Mathematical and Theoretical 46, 355204 (2013).

[63] C. Monthus, Journal of Statistical Mechanics: Theory and Experiment 2016, 073301 (2016).

[64] M. Serbyn, Z. Papić, and D. A. Abanin, Phys. Rev. B 96, 104201 (2017).

[65] A. D. Mirlin, Y. V. Fyodorov, F.-M. Dittes, J. Quezada, and T. H. Seligman, Phys. Rev. E 54, 3221 (1996).

[66] A. D. Mirlin, Physics Reports 326, 259 (2000).

[67] I. Varga and D. Braun, Phys. Rev. B 61, R11859 (2000).

[68] S. Welsh and D. E. Logan, arXiv:1806.01193 [cond-mat] (2018), arXiv: 1806.01193.

[69] D. E. Logan and S. Welsh, arXiv:1806.01688 [cond-mat] (2018), arXiv: 1806.01688.

[70] L. Rademaker and M. Ortuño, Phys. Rev. Lett. 116, 010404 (2016).

[71] C. Monthus, Journal of Physics A: Mathematical and Theoretical 49, 305002 (2016).

[72] S. J. Thomson and M. Schiró, Phys. Rev. B 97, 060201 (2018). 